\title{
Homogenization approximations for unidirectional transport past randomly distributed sinks
}

DOI:

10.1093/imamat/hxz004

\section{Document Version}

Accepted author manuscript

Link to publication record in Manchester Research Explorer

\section{Citation for published version (APA):}

Russell, M., \& Jensen, O. (2019). Homogenization approximations for unidirectional transport past randomly distributed sinks. IMA Journal of Applied Mathematics , 85(2), 231-262. https://doi.org/10.1093/imamat/hxz004

\section{Published in:}

IMA Journal of Applied Mathematics

\section{Citing this paper}

Please note that where the full-text provided on Manchester Research Explorer is the Author Accepted Manuscript or Proof version this may differ from the final Published version. If citing, it is advised that you check and use the publisher's definitive version.

\section{General rights}

Copyright and moral rights for the publications made accessible in the Research Explorer are retained by the authors and/or other copyright owners and it is a condition of accessing publications that users recognise and abide by the legal requirements associated with these rights.

\section{Takedown policy}

If you believe that this document breaches copyright please refer to the University of Manchester's Takedown Procedures [http://man.ac.uk/04Y6Bo] or contact uml.scholarlycommunications@manchester.ac.uk providing relevant details, so we can investigate your claim.

\section{OPEN ACCESS}


IMA Journal of Applied Mathematics (2019) Page 1 of 28

doi:10.1093/imamat/xxx000

\title{
Homogenization approximations for unidirectional transport past randomly distributed sinks
}

\author{
MatThew J. Russell \\ School of Mathematical Sciences, The University of Nottingham, Nottingham NG7 2RD, UK \\ OlIVER E. JENSEN \\ School of Mathematics, The University of Manchester, Manchester M13 9PL, UK
}

[Received on 21 January 2019]

\begin{abstract}
Transport in biological systems often occurs in complex spatial environments involving random structures. Motivated by such applications, we investigate an idealised model for solute transport past an array of point sinks, randomly distributed along a line, which remove solute via first-order kinetics. Random sink locations give rise to long-range spatial correlations in the solute field and influence the mean concentration. We present a non-standard approach to evaluating these features based on rationally approximating integrals of a suitable Green's function, which accommodates contributions varying on short and long lengthscales and has deterministic and stochastic components. We refine the results of classical two-scale methods for a periodic sink array (giving more accurate higher-order corrections with non-local contributions) and find explicit predictions for the fluctuations in concentration and disorderinduced corrections to the mean for both weakly and strongly disordered sink locations. Our predictions are validated across a large region of parameter space.
\end{abstract}

Keywords: homogenization; transport; spatial disorder

\section{Introduction}

Spatial disorder is intrinsic to many natural systems. In biomedical applications, for example, disorder needs careful consideration in developing constitutive models of heterogeneous multicellular tissues and it can influence transport processes in geometrically complex exchange organs such as the placenta and the lung. In practice, there may be only limited knowledge of the detailed structure of a tissue or organ of a given individual, which may simply be expressed in terms of statistics retrieved from a population of other individuals. If they are to support sound decision-making, theoretical models of transport or biomechanical function should account for such uncertainties, so that predictions can quantify the variability of outcomes within and between individuals. Geophysical applications, for example involving transport in subsurface porous media, raise many similar questions.

For materials with a multiscale structure (cells within a tissue, functional exchange units within an organ, pores within a rock, etc.), homogenization provides a powerful analytical tool, exemplified by the reduction of Stokes equations to Darcy's equation for porous medium transport (Burridge \& Keller, 1981; Rubinstein \& Torquato, 1989). For a strictly periodic medium, a variety of approaches (particularly asymptotic two-scale convergence and spatial averaging (Pavliotis \& Stuart, 2008; Davit et al., 2013)) yield leading-order approximations for the slowly-varying component of spatial fields, having well-studied convergence properties. Spatial fields for such problems are generally characterised by an almost-periodic variation at the microscale, modulated by slow variation over much longer lengthscales. Typically a unit cell problem at the microscale needs to be solved (or averaged) in order to derive an 
approximation capturing the macroscale variation. Such approaches can be extended to account for smooth variation in the properties of the unit cells over long lengthscales (Davit et al., 2013; Bruna \& Chapman, 2015). For materials with a random microstructure that lacks exact periodicity, however, less is understood about the accuracy of (deterministic) homogenization approximations, and leading-order approximations generally capture only mean behaviour. For proper uncertainty quantification, it is necessary to determine corrections that capture the stochastic variability in the quantity of interest. Such corrections are likely to be entangled with the discrete-to-continuous corrections of the classical homogenization approximation and are typically nonlocal (Heitzinger \& Ringhofer, 2014; Le Bris, 2014; Wood \& Valdés-Parada, 2013).

Maternal circulation in the placenta provides an interesting test-bed for such ideas (Jensen \& Chernyavsky, 2019). This organ exchanges dissolved gases, nutrients and other materials between maternal and fetal blood. Fetal blood vessels are arranged in tree-like structures called villi; maternal blood in the intervillous space flows past the outside of their branches, effectively passing through a disordered porous medium. Initial models described maternal blood flow using Darcy's equation (with a uniform permeability) in two spatial dimensions (Erian et al., 1977), with solute transport being described by a slowlyvarying (macroscale) advection/uptake equation with a uniform uptake coefficient (Chernyavsky et al., 2010). In an effort to understand the role of disorder in the arrangement of fetal vessels, Chernyavsky et al. $(2011,2012)$ studied simplified models of solute transport, reducing villous branches to point sinks (distributed along a line according to a prescribed distribution) and describing solute transport between sinks using a one-dimensional advection-diffusion equation. These studies tested the quality of the macroscale approximation in relation to simulations of the solute concentration under different sink realisations. Direct evaluation of the error (the homogenization residue) revealed its varying character across parameter space, its long-range covariance structure and its dependence on the statistical properties of the underlying sink distribution. In particular, parameter regimes were identified where the macroscale approximation fails because stochastic sink-to-sink variations in the solute concentration become dominant.

Chernyavsky et al. $(2011,2012)$ used an algebraic method to compute the homogenization residue directly, for zeroth-order uptake kinetics, which worked only over a limited range of parameter space. A more robust approach was presented by Russell et al. (2016), in a related problem assuming first-order kinetics and variable sink strength (rather than sink location). When disorder is weak, an expansion can be developed in which a deterministic periodic problem at leading order (which is readily homogenized) is perturbed to give a stochastic linear problem at the following order. Linearity allows the disorder due to individual sinks to be evaluated independently using a Green's function; the individual contributions are then assembled (exploiting the central limit theorem) to capture the overall disorder in the system, which has an inherently nonlocal structure. This method does not suffer the parameter-space restriction of earlier approaches, and it is developed further below. Taking the expansion to higher order, Russell et al. (2016) demonstrated how the macroscale approximation has a small but systematic error in the presence of weak disorder. The value of Green's functions in evaluating corrector fields was demonstrated also by Wood \& Valdés-Parada (2013) and Heitzinger \& Ringhofer (2014); the latter authors for example considered a Poisson problem with a distributed source that is statistically uncorrelated over a periodic array of cells.

In the present study we consider how the random spatial location (along a line) of identical first-order sinks influences the distribution of a solute that moves between them by advection and diffusion. We consider periodic, weakly disordered and strongly disordered sink locations. In the periodic case, we use a Green's function approach (instead of the traditional two-scale expansion, which relies on an ad hoc periodicity assumption) to derive the macroscale solution and its corrections. The methods deviate in 
their predictions at sub-leading-order: we demonstrate numerically that the Green's function approach is more accurate than the classical approach. To address disorder, we construct an empirical expansion about the macroscale solution, again exploiting Green's functions, correcting successively for the discrete-to-continuous and periodic-to-disordered effects. The expansion is shown to be effective both in the weakly disordered limit (as demonstrated in Russell et al. 2016) and the strongly disordered case (when sinks are distributed uniformly randomly in a finite interval). We do not attempt to provide rigorous convergence proofs; however having adopted a non-standard expansion, we apply careful asymptotic techniques in order to approximate the sums and integrals that arise and to establish their relative magnitudes.

\section{Model definition}

We model steady transport of a solute past a linear array of point sinks using an advection-diffusionuptake equation. The sinks have first-order uptake kinetics and sit at discrete locations $x^{*}=\xi_{j}^{*}, j=$ $1, \ldots, N$; see Figure 1(a). We introduce the sink density, Péclet number, Damköhler number and concentration scale as

$$
\varepsilon=\frac{\ell}{L}=\frac{1}{N+1}, \quad \mathrm{Pe}=\frac{U \ell}{D}, \quad \mathrm{Da}=\frac{S_{0} \ell}{D}, \quad C_{0}=\frac{q L}{D},
$$

respectively, where $\ell$ is the average inter-sink distance, $L$ is the domain length, $U$ is the advection velocity, $D$ is the diffusion coefficient, $S_{0}$ is the sink strength per unit concentration and $q$ is the flux at the inlet boundary. At the downstream boundary we impose zero concentration, $\left.C^{*}\right|_{x^{*}=L}=0$. The Péclet number Pe represents the strength of advection relative to diffusion and the Damköhler number Da represents the strength of uptake relative to diffusion. The governing equation and boundary conditions for the solute concentration $C^{*}\left(x^{*}\right)$ are

$$
\begin{gathered}
D C_{x^{*} x^{*}}^{*}-U C_{x^{*}}^{*}=S_{0} C^{*}\left(x^{*}\right) \sum_{j=1}^{N} \delta\left(x^{*}-\xi_{j}^{*}\right), \quad 0 \leqslant x^{*} \leqslant L, \\
\left.U C^{*}\right|_{x^{*}=0}-\left.D C_{x^{*}}^{*}\right|_{x^{*}=0}=q,\left.\quad C^{*}\right|_{x^{*}=L}=0 .
\end{gathered}
$$

Introducing the dimensionless variables

$$
x=x^{*} / \ell, \quad \xi_{j}=\xi_{j}^{*} / \ell, \quad C(x)=C^{*}\left(x^{*}\right) / C_{0},
$$

(2.2) becomes,

$$
\begin{gathered}
C_{x x}-\operatorname{Pe} C_{x}=\operatorname{Da} C(x) \sum_{j=1}^{N} \delta\left(x-\xi_{j}\right), \quad 0 \leqslant x \leqslant \varepsilon^{-1}, \\
\left.\operatorname{Pe} C\right|_{0}-\left.C_{x}\right|_{0}=\varepsilon,\left.\quad C\right|_{\mathcal{E}^{-1}}=0 .
\end{gathered}
$$

For later convenience, we set $\xi_{0}=0$ and $\xi_{N+1}=\varepsilon^{-1}$. Integrating over the whole domain yields the overall flux balance

$$
\varepsilon+\left.C_{x}\right|_{\varepsilon^{-1}}=\mathrm{Da} \sum_{j=1}^{N} C\left(\xi_{j}\right)
$$

which provides a direct method of determining the net uptake by the internal sinks. We will consider the following sink distributions: periodically-located, $\xi_{j}=j$; normally-perturbed from a periodic arrangement, $\xi_{j} \sim \mathscr{N}\left(j, \sigma^{2}\right)$ for some small variance $\sigma^{2}$; and uniformly distributed in the domain according to 
$\mathscr{U}\left(0, \varepsilon^{-1}\right)$, sorted into ascending order. In the uniformly-random case, the sink locations $\xi_{j}$ are order statistics of the uniform distribution and are then spatially correlated, unlike the independently drawn uniform variates.

The problem described by (2.4) involves a number of spatial scales (e.g. domain length and sinkto-sink distance) over which advection, diffusion and uptake take place. Spatial disorder, in the form of random sink locations, adds additional complexity to the problem. These features are illustrated in Figure 1(b), which shows $10^{3}$ concentration profiles generated numerically using the method described in Appendix A. Each realisation has $N=49$ sinks whose locations are uniformly randomly distributed across the domain. The parameters are $\mathrm{Pe}=1, \mathrm{Da}=\frac{1}{2} \varepsilon^{1 / 2}$, which is a regime with strong advection and uptake characterised by a prominent sink-to-sink "staircase" structure and uptake across a large portion of the domain. We seek to characterise the mean and (co)variance of the concentration across the domain.

\section{Constructing an expansion}

We introduce the linear differential operator $\mathscr{L}=\partial_{x}^{2}-\operatorname{Pe} \partial_{x}-$ Da and boundary conditions $\mathscr{B} C=$ $\left\{\left.\left(\mathrm{Pe}-\partial_{x}\right) C\right|_{0},\left.C\right|_{\varepsilon^{-1}}\right\}$, and let $C_{\mathrm{H}}(x)$ satisfy the homogenized analogue of (2.4), in which point sinks are replaced by a smoothly varying term,

$$
\mathscr{L} C_{\mathrm{H}}=0, \quad \mathscr{B} C_{\mathrm{H}}=\{\varepsilon, 0\}, \quad 0 \leqslant x \leqslant \varepsilon^{-1} .
$$

Defining $\phi \equiv \sqrt{\mathrm{Da}+\mathrm{Pe}^{2} / 4}$ and $g(x) \equiv \mathrm{Pe} \sinh (\phi x)+2 \phi \cosh (\phi x)$, (3.1) has the exact solution

$$
C_{\mathrm{H}}(x)=\frac{2 \varepsilon}{g\left(\varepsilon^{-1}\right)} e^{\frac{1}{2} \operatorname{Pe} x} \sinh \left(\phi\left[\varepsilon^{-1}-x\right]\right), \quad 0 \leqslant x \leqslant \varepsilon^{-1} .
$$

$C_{\mathrm{H}}$ represents the leading-order homogenized solution to (2.4) as $\varepsilon \rightarrow 0$ for $\mathrm{Pe}=O(\varepsilon), \mathrm{Da}=O\left(\varepsilon^{2}\right)$, when the sinks are distributed periodically; see Russell et al. (2016) and Appendix B, where we revisit the classical two-scale expansion for this problem. We will initially work in this parameter regime, for which there is a dominant balance between advection, diffusion and uptake across the domain. However, unlike the classical approach, we make no assumption in what follows about $C$ depending on independent long- and short-range variables.

The Green's function $G(x, y)$ associated with $\mathscr{L}$ under homogeneous boundary conditions $\mathscr{B} G=$ $\{0,0\}$ satisfies $\mathscr{L} G=\delta(x-y)$. We write

$$
G(x, y)= \begin{cases}G^{-}(x, y), & 0 \leqslant x \leqslant y \leqslant \varepsilon^{-1} \\ G^{+}(x, y), & 0 \leqslant y \leqslant x \leqslant \varepsilon^{-1} .\end{cases}
$$

$G(x, y)$ is piecewise smooth, continuous at $x=y$ and satisfies the following jump conditions, resulting from the point source at $x=y$ :

$$
G_{x}^{+}(y, y)-G_{x}^{-}(y, y)=1, \quad G_{y}^{-}(x, x)-G_{y}^{+}(x, x)=1 .
$$

The two pieces of the Green's function can be expressed as

$$
\begin{aligned}
& G^{-}(x, y)=\frac{g(x)}{\phi g\left(\varepsilon^{-1}\right)} e^{\frac{1}{2} \operatorname{Pe}(x-y)} \sinh \left(\phi\left[y-\varepsilon^{-1}\right]\right), \\
& G^{+}(x, y)=e^{\operatorname{Pe}(x-y)} G^{-}(y, x) .
\end{aligned}
$$


(a)

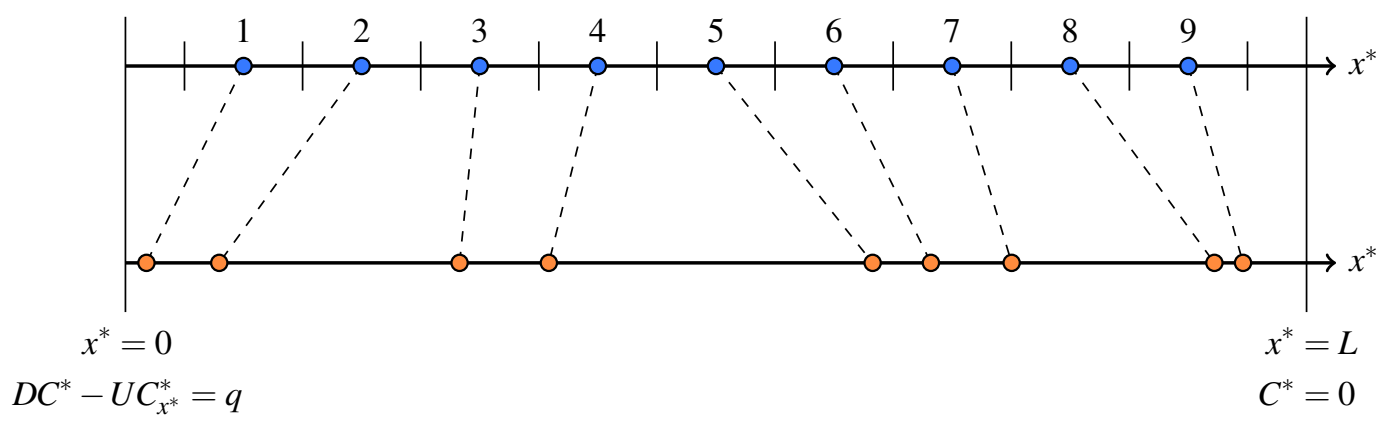

(b)

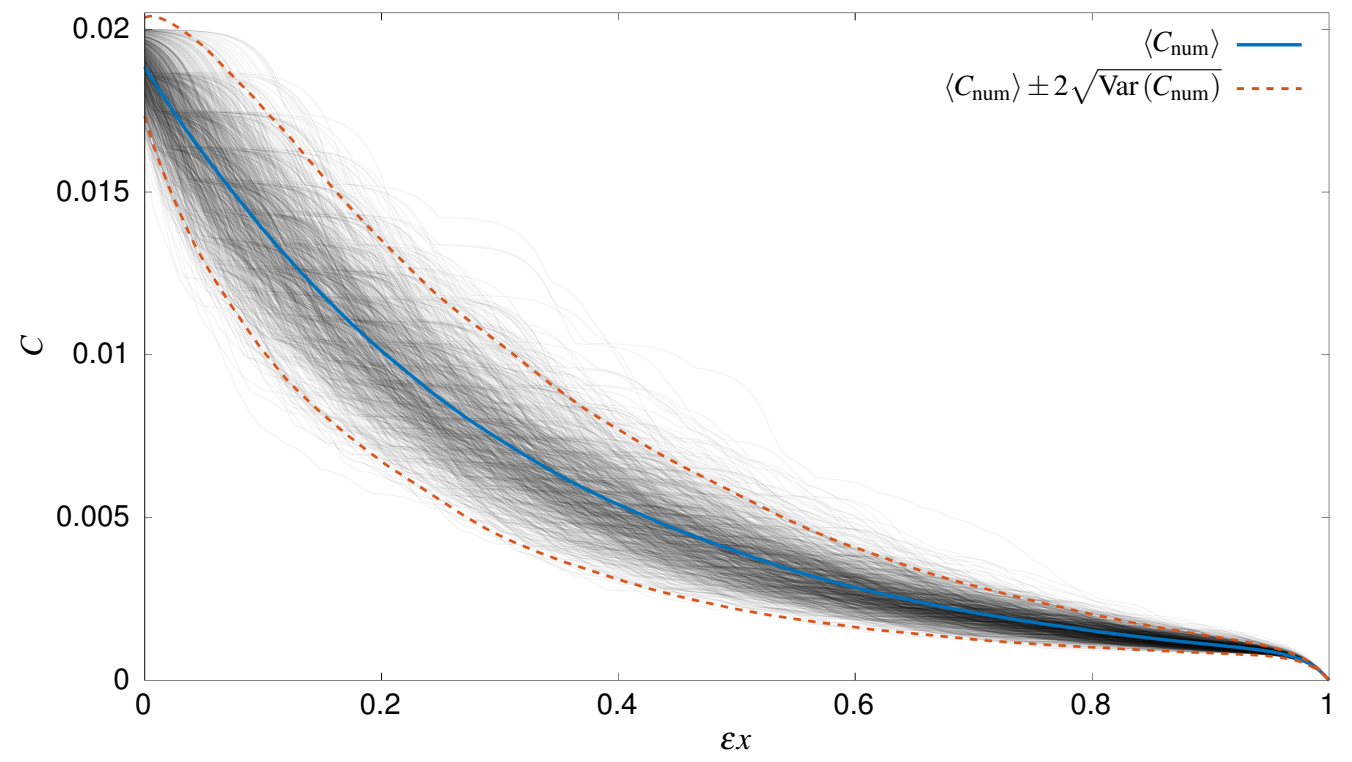

FIG. 1. (a) Diagram of the problem domain, with inlet and outlet conditions indicated below their respective boundaries. On the top axis, a periodic configuration of $N=9$ sinks is shown. Blue circles mark the sink locations and vertical lines delimit the associated unit cells. The bottom axis shows one realisation of a random distribution of sinks, denoted by orange circles. The dashed lines indicate how we label sinks in ascending order, regardless of how they were drawn from the distribution. (b) An ensemble of $10^{3}$ concentration profiles (thin black lines). Each realisation has $N=49$ sinks (i.e. $\varepsilon=0.02$ ) with uniformly random locations, and $\mathrm{Pe}=1, \mathrm{Da}=\frac{1}{2} \varepsilon^{1 / 2}$. Also shown is the sample mean (solid blue) and two standard deviations either side of the mean (dashed orange).

Later we will use the identity

$$
C_{\mathrm{H}}(x)=-\varepsilon G^{+}(x, 0) .
$$

Figure 2 compares the leading-order approximation $C_{\mathrm{H}}$ with numerical solutions of (2.4) (obtained using the method described in Appendix A for $N=99$ periodically located sinks and a range of (Pe, Da)-values, showing good agreement. Also shown as insets in each panel are illustrative plots of the Green's function (3.3). 
(a) $\mathrm{Pe}=\varepsilon^{2}, \mathrm{Da}=\varepsilon$

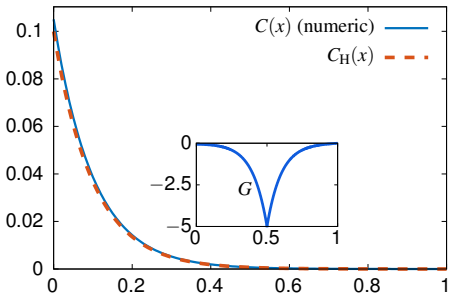

(d) $\mathrm{Pe}=\varepsilon^{2}, \mathrm{Da}=\varepsilon^{2}$

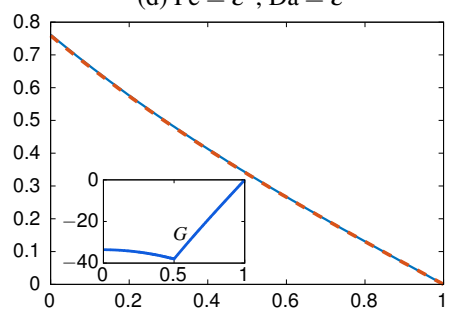

(g) $\mathrm{Pe}=\varepsilon^{2}, \mathrm{Da}=\varepsilon^{3}$

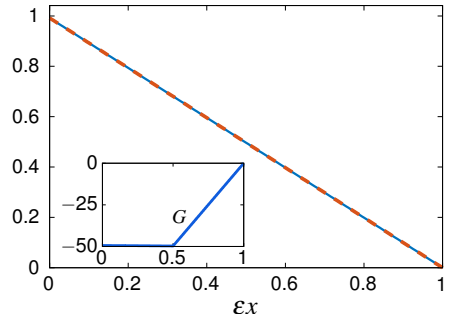

(b) $\mathrm{Pe}=\varepsilon, \mathrm{Da}=\varepsilon$

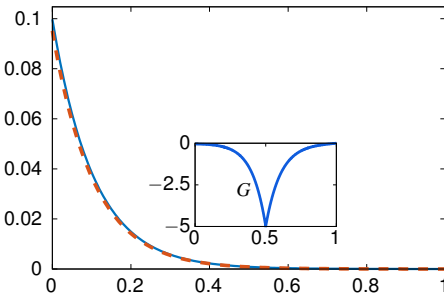

(e) $\mathrm{Pe}=\varepsilon, \mathrm{Da}=\varepsilon^{2}$

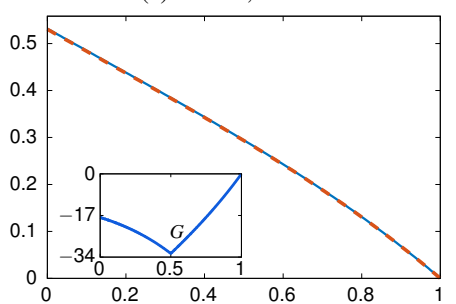

(h) $\mathrm{Pe}=\varepsilon, \mathrm{Da}=\varepsilon^{3}$

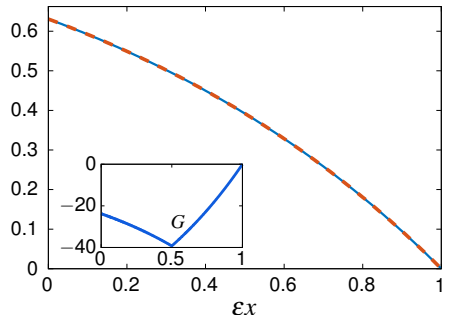

(c) $\mathrm{Pe}=1, \mathrm{Da}=\varepsilon$

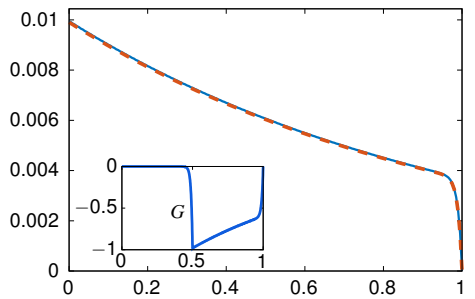

(f) $\mathrm{Pe}=1, \mathrm{Da}=\varepsilon^{2}$

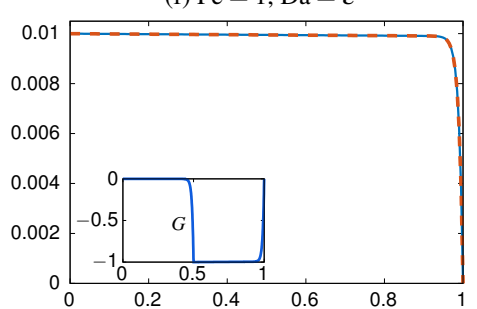

(i) $\mathrm{Pe}=1, \mathrm{Da}=\varepsilon^{3}$

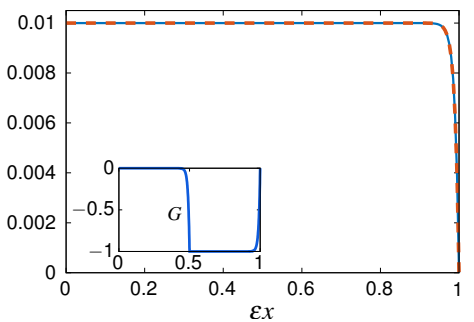

FIG. 2. Comparison between the numerical solution of (2.4) $C(x)$ with a periodic sink array (solid blue) and the leading-order homogenized approximation $C_{\mathrm{H}}$ satisfying (3.2) (dashed orange), both plotted versus $\varepsilon x$. Insets show the corresponding Green's function (3.3) with the point source located in the centre of the domain, $y=\frac{1}{2} \varepsilon^{-1}$ for illustrative purposes. There are $N=99$ sinks in all cases, so $\varepsilon=10^{-2}$, and the Pe, Da values are indicated in the panel titles, with all combinations of $\mathrm{Pe} \in\left\{\varepsilon^{2}, \varepsilon, 1\right\}$, $\mathrm{Da} \in\left\{\varepsilon^{3}, \varepsilon^{2}, \varepsilon\right\}$ shown.

Inspection of (3.2) and (3.5) reveals that $C_{\mathrm{H}}(x)$ and $\varepsilon G(x, y)$ vary by $O(1)$ as $x, y$ vary across the domain (that is as $\varepsilon x$, $\varepsilon y$ vary by $O(1)$ ), in the distinguished limit $\mathrm{Pe}=O(\varepsilon), \mathrm{Da}=O\left(\varepsilon^{2}\right)$. This can be seen in Figure 2(e). Increasing uptake relative to diffusion, characterised by elevated Da, leads to more rapid decay near the inlet (see Figure $2 b$ ); increasing advection relative to diffusion, characterised by elevated Pe, leads to a diffusive boundary layer near the outlet and, for $G$, near $x=y$ (see Figure 2f). We introduce the notation

$$
\left.\left(G C_{\mathrm{H}}\right)\right|_{x, y} \equiv G(x, y) C_{\mathrm{H}}(y)
$$

and use the corresponding notation with $G^{+}$and $G^{-}$in place of $G$, noting that for $\mathrm{Pe}=O(\varepsilon), \mathrm{Da}=$ $O\left(\varepsilon^{2}\right)$, each derivative of $C_{\mathrm{H}}$ and $G$ with respect to $x$ or $y$ reduces its magnitude by $O(\varepsilon)$.

To account for the discrete nature of the sinks and their spatial distribution in the domain, we pose an expansion for solutions to (2.4) consisting of the approximation (3.2) and a series of correction terms. 
We therefore write $C=C_{\mathrm{H}}+\hat{C}$, where the corrections $\hat{C}$ satisfy $\mathscr{B} \hat{C}=\{0,0\}$ and

$$
\begin{aligned}
\mathscr{L} \hat{C}=\operatorname{Da} & \left\{C_{\mathrm{H}}(x)\left[\sum_{j=1}^{N} \delta(x-j)-1\right]+C_{\mathrm{H}}(x) \sum_{j=1}^{N}\left[\delta\left(x-\xi_{j}\right)-\delta(x-j)\right]\right. \\
+ & \left.\hat{C}(x)\left[\sum_{j=1}^{N} \delta(x-j)-1\right]+\hat{C}(x) \sum_{j=1}^{N}\left[\delta\left(x-\xi_{j}\right)-\delta(x-j)\right]\right\} .
\end{aligned}
$$

Since (3.8) is linear, we may consider the solution of the sub-problems associated with each right-hand sum separately. The first is

$$
\mathscr{L} \hat{C}_{a}=\operatorname{Da} C_{\mathrm{H}}(x)\left[\sum_{j=1}^{N} \delta(x-j)-1\right], \quad \mathscr{B} \hat{C}_{a}=\{0,0\},
$$

which describes a transport problem modelling the difference between a periodic sink arrangement and a smooth sink distribution, with strengths modulated by the leading-order concentration profile $C_{\mathrm{H}}$. Using the Green's function (3.3) we can write the solution as

$$
\hat{C}_{a}(x)=\left.\mathrm{Da} \int_{0}^{\varepsilon^{-1}}\left(G C_{\mathrm{H}}\right)\right|_{x, y}\left[\sum_{j=1}^{N} \delta(y-j)-1\right] \mathrm{d} y .
$$

We expect $\hat{C}_{a}$ to provide the dominant corrections due to discrete-sink effects to the leading-order solution $C_{\mathrm{H}}$. Similarly, the second sub-problem from (3.8) is

$$
\mathscr{L} \hat{C}_{b}=\operatorname{Da} C_{\mathrm{H}}(x) \sum_{j=1}^{N}\left[\delta\left(x-\xi_{j}\right)-\delta(x-j)\right], \quad \mathscr{B} \hat{C}_{b}=\{0,0\},
$$

so that

$$
\begin{aligned}
\hat{C}_{b}(x) & =\left.\mathrm{Da} \int_{0}^{\mathcal{E}^{-1}}\left(G C_{\mathrm{H}}\right)\right|_{x, y} \sum_{j=1}^{N}\left[\delta\left(y-\xi_{j}\right)-\delta(y-j)\right] \mathrm{d} y \\
& =\mathrm{Da} \sum_{j=1}^{N}\left[\left.\left(G C_{\mathrm{H}}\right)\right|_{x, \xi_{j}}-\left.\left(G C_{\mathrm{H}}\right)\right|_{x, j}\right],
\end{aligned}
$$

which captures the effects of displacing sinks from a periodic to a disordered arrangement, with strengths again given by $C_{\mathrm{H}}$. We may recursively continue the expansion in the following way

$$
C=C_{\mathrm{H}}+\left(\hat{C}_{a}+\hat{C}_{b}\right)+\left(\hat{C}_{a a}+\hat{C}_{a b}+\hat{C}_{b a}+\hat{C}_{b b}\right)+\cdots
$$

(a form of Duhamel expansion (Bal, 2011)) where the remaining subproblems in (3.8) become

$$
\begin{array}{ll}
\mathscr{L} \hat{C}_{a a}=\operatorname{Da} \hat{C}_{a}\left[\sum_{j=1}^{N} \delta(x-j)-1\right], & \mathscr{L} \hat{C}_{a b}=\operatorname{Da} \hat{C}_{a} \sum_{j=1}^{N}\left[\delta\left(x-\xi_{j}\right)-\delta(x-j)\right] \\
\mathscr{L} \hat{C}_{b a}=\operatorname{Da} \hat{C}_{b}\left[\sum_{j=1}^{N} \delta(x-j)-1\right], & \mathscr{L} \hat{C}_{b b}=\operatorname{Da} \hat{C}_{b} \sum_{j=1}^{N}\left[\delta\left(x-\xi_{j}\right)-\delta(x-j)\right],
\end{array}
$$


etc., each with homogeneous boundary conditions. Each term with a subscript containing one or more $b$ involves a random variable. The problem of a deterministic, periodic sink array is fully described by $C_{\mathrm{H}}, \hat{C}_{a}, \hat{C}_{a a}, \ldots$ This series is not assumed to be asymptotic in general, but the size of each term can be estimated a posteriori based on their dependence on $C_{\mathrm{H}}, G$ and the parameters Da and Pe to suggest an asymptotic reordering of terms and to assess convergence. Magnitudes of the remaining terms will depend on the choice of sink distribution so we must analyse each case separately.

The following strategy will be used to estimate magnitudes in terms of $\varepsilon$ in the distinguished limit $\mathrm{Pe}=O(\varepsilon), \mathrm{Da}=O\left(\varepsilon^{2}\right)$, for which $C_{\mathrm{H}}$ and $G$ are piecewise smooth on the macroscale (Figure 2e). In this limit sums, perhaps with finitely many terms excluded, and integrals over the domain will contribute a factor of $\varepsilon^{-1} \approx N$; $G$ contributes a factor of $\varepsilon^{-1}$ and $C_{\mathrm{H}}$ a factor of 1 ; each successive derivative of $G$ and $C_{\mathrm{H}}$ with respect to $x$ or $y$ gains an additional factor $\varepsilon$ in magnitude, reflecting the slow variation of these functions across the domain (see Figure 2e). Alternative scaling arguments will be required in other parameter regimes.

\section{The periodic problem}

\subsection{Solving for $\hat{C}_{a}$}

To solve (3.9) for $\hat{C}_{a}$, we split the domain $\left[0, \varepsilon^{-1}\right]$ into unit cells $\left(j-\frac{1}{2}, j+\frac{1}{2}\right)$ for $j=1,2, \ldots, N$, and two half-cells $\left[0, \frac{1}{2}\right)$ and $\left(\varepsilon^{-1}-\frac{1}{2}, \varepsilon^{-1}\right]$ at the ends. The cell which contains $x$, say $j=k$ where $k \equiv\left\lfloor x+\frac{1}{2}\right\rfloor$, is treated separately and we split the integral at $y=x$ to allow for careful handling of discontinuities. We Taylor expand $\left.\left(G C_{\mathrm{H}}\right)\right|_{x, y}$ inside the integral around $y=j$ for $j \neq k$, around $y= \pm x$ for $j=k$, and around $y=0$ and $y=\varepsilon^{-1}$ for the inlet and outlet half-cells, respectively. Then, (3.10) becomes

$$
\begin{aligned}
\hat{C}_{a}(x)=\mathrm{Da} & \left\{\sum_{j \neq k} \int_{j-1 / 2}^{j+1 / 2}\left[\left.\left(G C_{\mathrm{H}}\right)\right|_{x, j}+\left.(y-j)\left(G C_{\mathrm{H}}\right)_{y}\right|_{x, j}+\left.\frac{1}{2}(y-j)^{2}\left(G C_{\mathrm{H}}\right)_{y y}\right|_{x, j}+\cdots\right][\delta(y-j)-1] \mathrm{d} y\right. \\
& +\int_{k-1 / 2}^{x}\left[\left.\left(G^{+} C_{\mathrm{H}}\right)\right|_{x, x}+\left.(y-x)\left(G^{+} C_{\mathrm{H}}\right)_{y}\right|_{x, x}+\left.\frac{1}{2}(y-x)^{2}\left(G^{+} C_{\mathrm{H}}\right)_{y y}\right|_{x, x}+\cdots\right][\delta(y-k)-1] \mathrm{d} y \\
& +\int_{x}^{k+1 / 2}\left[\left.\left(G^{-} C_{\mathrm{H}}\right)\right|_{x, x}+\left.(y-x)\left(G^{-} C_{\mathrm{H}}\right)_{y}\right|_{x, x}+\left.\frac{1}{2}(y-x)^{2}\left(G^{-} C_{\mathrm{H}}\right)_{y y}\right|_{x, x}+\cdots\right][\delta(y-k)-1] \mathrm{d} y \\
& -\int_{0}^{1 / 2}\left[\left.\left(G C_{\mathrm{H}}\right)\right|_{x, 0}+\left.y\left(G C_{\mathrm{H}}\right)_{y}\right|_{x, 0}+\left.\frac{1}{2} y^{2}\left(G C_{\mathrm{H}}\right)_{y y}\right|_{x, 0}+\cdots\right] \mathrm{d} y \\
& \left.-\int_{\mathcal{\varepsilon}^{-1}-1 / 2}^{\varepsilon^{-1}}\left[\left.\left(G C_{\mathrm{H}}\right)\right|_{x, \varepsilon^{-1}}+\left.\left(y-\varepsilon^{-1}\right)\left(G C_{\mathrm{H}}\right)_{y}\right|_{x, \varepsilon^{-1}}+\left.\frac{1}{2}\left(y-\varepsilon^{-1}\right)^{2}\left(G C_{\mathrm{H}}\right)_{y y}\right|_{x, \varepsilon^{-1}}+\cdots\right] \mathrm{d} y\right\} .
\end{aligned}
$$

This expansion of $\left(G C_{\mathrm{H}}\right)$ reduces each integrand to a sum of polynomials, each multiplied by $[\delta(x-$ $j)-1]$ or similar (except for the half-cell integrals). Integrating over the two half-cells,

$$
\begin{aligned}
-\int_{0}^{1 / 2}[\cdots] \mathrm{d} y & =-\left.\frac{1}{2}\left(G^{+} C_{\mathrm{H}}\right)\right|_{x, 0}-\left.\frac{1}{8}\left(G^{+} C_{\mathrm{H}}\right)_{y}\right|_{x, 0}-\left.\frac{1}{48}\left(G^{+} C_{\mathrm{H}}\right)_{y y}\right|_{x, 0}+\cdots, \\
-\int_{\mathcal{E}^{-1}-1 / 2}^{\mathcal{E}^{-1}}[\cdots] \mathrm{d} y & =-\left.\frac{1}{24}\left(G_{y}^{-} C_{\mathrm{H} y}\right)\right|_{x, \mathcal{E}^{-1}}+\cdots
\end{aligned}
$$

where we have eliminated terms using the boundary conditions (3.1). The first two terms of the first integral in (4.1) vanish (see Appendix C), as does the first term of the integrals over cell $j=k$, for which 
we also use that $\left.G^{+}\right|_{x, x}=\left.G^{-}\right|_{x, x}$. We use the identities in Appendix $\mathrm{C}$ again to calculate the remaining integrals, and

$$
\begin{aligned}
\hat{C}_{a}(x)=\mathrm{Da}\{ & -\left.\frac{1}{24} \sum_{j \neq k}\left(G C_{\mathrm{H}}\right)_{y y}\right|_{x, j}-\left.\frac{1}{24}\left(G C_{\mathrm{H} y y}\right)\right|_{x, x} \\
& +\left.\left(G_{y}^{+} C_{\mathrm{H}}\right)\right|_{x, x}\left[(k-x) H(x-k)+\frac{1}{2}\left(x-k+\frac{1}{2}\right)^{2}\right] \\
& +\left.\left(G_{y}^{-} C_{\mathrm{H}}\right)\right|_{x, x}\left[(k-x) H(k-x)-\frac{1}{2}\left(x-k-\frac{1}{2}\right)^{2}\right] \\
& +\left.\frac{1}{2}\left(G_{y y}^{+} C_{\mathrm{H}}+2 G_{y}^{+} C_{\mathrm{H} y}\right)\right|_{x, x}\left[(k-x)^{2} H(x-k)-\frac{1}{3}\left(x-k+\frac{1}{2}\right)^{3}\right] \\
& +\left.\frac{1}{2}\left(G_{y y}^{-} C_{\mathrm{H}}+2 G_{y}^{-} C_{\mathrm{H} y}\right)\right|_{x, x}\left[(k-x)^{2} H(k-x)+\frac{1}{3}\left(x-k-\frac{1}{2}\right)^{3}\right] \\
& \left.-\left.\frac{1}{2}\left(G^{+} C_{\mathrm{H}}\right)\right|_{x, 0}-\left.\frac{1}{8}\left(G^{+} C_{\mathrm{H}}\right)_{y}\right|_{x, 0}-\left.\frac{1}{48}\left(G^{+} C_{\mathrm{H}}\right)_{y y}\right|_{x, 0}-\left.\frac{1}{24}\left(G_{y}^{-} C_{\mathrm{H} y}\right)\right|_{x, \varepsilon^{-1}}+\cdots\right\} .
\end{aligned}
$$

Using the jump conditions (3.4), we write $G_{y}^{-}$in terms of $G_{y}^{+}$to give

$$
\begin{aligned}
\hat{C}_{a}(x)=\mathrm{Da}\{ & -\left.\frac{1}{24} \sum_{j \neq k}\left(G C_{\mathrm{H}}\right)_{y y}\right|_{x, j}-\left.\frac{1}{24}\left(G C_{\mathrm{H} y y}\right)\right|_{x, x}-\left.\frac{1}{12}\left(G_{y}^{+} C_{\mathrm{H} y}\right)\right|_{x, x} \\
& +C_{\mathrm{H}}(x)\left[(k-x) H(k-x)-\frac{1}{2}\left(x-k-\frac{1}{2}\right)^{2}\right] \\
& +C_{\mathrm{H} y}(x)\left[(k-x)^{2} H(k-x)+\frac{1}{3}\left(x-k-\frac{1}{2}\right)^{3}\right] \\
& +\left.\frac{1}{2}\left(G_{y y}^{+} C_{\mathrm{H}}\right)\right|_{x, x}\left[(k-x)^{2} H(x-k)-\frac{1}{3}\left(x-k+\frac{1}{2}\right)^{3}\right] \\
& +\left.\frac{1}{2}\left(G_{y y}^{-} C_{\mathrm{H}}\right)\right|_{x, x}\left[(k-x)^{2} H(k-x)+\frac{1}{3}\left(x-k-\frac{1}{2}\right)^{3}\right] \\
& \left.-\left.\frac{1}{2}\left(G^{+} C_{\mathrm{H}}\right)\right|_{x, 0}-\left.\frac{1}{8}\left(G^{+} C_{\mathrm{H}}\right)_{y}\right|_{x, 0}-\left.\frac{1}{48}\left(G^{+} C_{\mathrm{H}}\right)_{y y}\right|_{x, 0}-\left.\frac{1}{24}\left(G_{y}^{-} C_{\mathrm{H} y}\right)\right|_{x, \varepsilon^{-1}}+\cdots\right\} .
\end{aligned}
$$

Recalling that $G=O\left(\varepsilon^{-1}\right)$ and $C_{\mathrm{H}}=O(1)$ when $\mathrm{Pe}=O(\varepsilon), \mathrm{Da}=O\left(\varepsilon^{2}\right)$, we can collect the terms in (4.2) by magnitude:

$$
\begin{gathered}
\hat{C}_{a}(x)=\mathrm{Da}\left\{-\left.\frac{1}{2}\left(G^{+} C_{\mathrm{H}}\right)\right|_{x, 0}-\left.\frac{1}{8}\left(G^{+} C_{\mathrm{H}}\right)_{y}\right|_{x, 0}+C_{\mathrm{H}}(x)\left[(k-x) H(k-x)-\frac{1}{2}\left(x-k-\frac{1}{2}\right)^{2}\right]\right. \\
\left.-\left.\frac{1}{24} \sum_{j \neq k}\left(G C_{\mathrm{H}}\right)_{y y}\right|_{x, j}+O(\varepsilon)\right\} .
\end{gathered}
$$

Using (D.4) to approximate sums with integrals and using the jump condition (3.4), gives

$$
-\left.\frac{1}{24} \sum_{j \neq k}\left(G C_{\mathrm{H}}\right)_{y y}\right|_{x, j}=\left.\frac{1}{24}\left(G C_{\mathrm{H}}\right)_{y}\right|_{x, 0}+\frac{1}{24} C_{\mathrm{H}}(x)+O(\varepsilon) .
$$

Defining $f(x) \equiv-x H(-x)-\frac{1}{2}\left(x-\frac{1}{2}\right)^{2}+\frac{1}{24}$, for $|x|<1 / 2$ (and zero otherwise), so that $f$ has zero unit cell average $\int_{-1 / 2}^{1 / 2} f(x) \mathrm{d} x=0$, it follows that

$$
\hat{C}_{a}(x)=\mathrm{Da}\left[\frac{1}{2} \varepsilon^{-1} C_{\mathrm{H}}(0) C_{\mathrm{H}}(x)+\frac{1}{12} \varepsilon^{-1} C_{\mathrm{H} y}(0) C_{\mathrm{H}}(x)+f(x-k) C_{\mathrm{H}}(x)+O(\varepsilon)\right], \quad k=\left\lfloor x+\frac{1}{2}\right\rfloor .
$$

The term $f(x-k) C_{\mathrm{H}}(x)$ in (4.4) varies rapidly on the scale of individual unit cells, modulated on an $O\left(\varepsilon^{-1}\right)$ lengthscale by $C_{\mathrm{H}}$. The two additional contributions are slowly varying. In the distinguished 
10 of 28

limit, the slowly varying terms have magnitudes $O(\varepsilon)$ and $O\left(\varepsilon^{2}\right)$ respectively and the oscillatory term has magnitude $O\left(\varepsilon^{2}\right)$. The neglected terms are of magnitude $O\left(\varepsilon^{3}\right)$; however, we show below that $\hat{C}_{a a}$ also contributes at $O\left(\varepsilon^{2}\right)$.

\subsection{Solving for $\hat{C}_{a a}$}

With periodically located sinks, the next non-zero term in the expansion (3.13) is $\hat{C}_{a a}$, satisfying (3.14). Recognising the recursive nature of the expansion, $\hat{C}_{a a}$ takes the same form as $\hat{C}_{a}$, but with the role of $C_{\mathrm{H}}$ fulfilled by $\hat{C}_{a}$ as follows,

$$
\hat{C}_{a a}(x)=\operatorname{Da}\left[-\frac{1}{2} G^{+}(x, 0) \hat{C}_{a}(0)-\left.\frac{1}{12}\left(G^{+}(x, y) \hat{C}_{a}(y)\right)_{y}\right|_{y=0}+f(x-k) \hat{C}_{a}(x)+\cdots\right] .
$$

Retaining only the leading-order, slowly varying terms in $\hat{C}_{a}$, gives

$$
\hat{C}_{a a}(x)=\mathrm{Da}^{2} \varepsilon^{-2}\left[\frac{1}{4}\left(C_{\mathrm{H}}(0)\right)^{2} C_{\mathrm{H}}(x)+\cdots\right] .
$$

At sub-leading orders $\hat{C}_{a a}$ contains oscillatory terms from $\hat{C}_{a}$ and further terms arising from the $f(x-$ k) $\hat{C}_{a}(x)$ term in (4.5).

In the distinguished limit, $\hat{C}_{a a}$ has magnitude $O\left(\varepsilon^{2}\right)$ so must be included in an expansion of the concentration up to this order. The next correction, $\hat{C}_{a a a}$, is related to $\hat{C}_{a a}$ in the same way that $\hat{C}_{a a}$ is related to $\hat{C}_{a}$ in (4.5). The largest terms in $\hat{C}_{a a a}$ are therefore $O\left(\varepsilon^{3}\right)$, and since this recursive pattern continues for the higher corrections we conclude that all contributions up to $O\left(\varepsilon^{2}\right)$ are captured by the corrections up to $\hat{C}_{a a}$. Interestingly, our prediction of the sub-leading-order terms for the periodicallylocated sinks problem contain extra terms up to order $O\left(\varepsilon^{2}\right)$ compared with results from classical twoscale homogenization; see Appendix B for a derivation of the classical results.

For the purposes of comparing the theoretical predictions with simulations, we define the following residual (using leading-order expressions for $\hat{C}_{a}$ and $\hat{C}_{a a}$ )

$$
\begin{aligned}
r(x) & \equiv C_{\mathrm{num}}(x)-\left(C_{\mathrm{H}}(x)+\left[\hat{C}_{a}(x)-\mathrm{Da} f(x-k) C_{\mathrm{H}}(x)\right]+\hat{C}_{a a}(x)\right) \\
& =C_{\mathrm{num}}(x)-C_{\mathrm{H}}(x)\left(1+\frac{1}{2} \mathrm{Da} \varepsilon^{-1}\left[C_{\mathrm{H}}(0)+\frac{1}{6} C_{\mathrm{H} y}(0)\right]+\frac{1}{4} \mathrm{Da}^{2} \varepsilon^{-2}\left[C_{\mathrm{H}}(0)\right]^{2}\right),
\end{aligned}
$$

in which we have subtracted from numerical solutions $C_{\text {num }}(x)$ of the full problem (2.4) all the terms in $\hat{C}_{a}$ and $\hat{C}_{a a}$ appearing in $(4.4,4.6)$ which vary slowly across the domain, plus of course $C_{\mathrm{H}}$. What remains, to leading-order, is the numerical prediction of the sink-to-sink oscillating part of the solution. We further define a residual based on the results of the classical method in Appendix B, which to the same level of accuracy is

$$
r_{\mathrm{c}}(x) \equiv C_{\mathrm{num}}(x)-C_{\mathrm{H}}(x)\left(1+\frac{1}{2} \mathrm{Da}^{-1} C_{\mathrm{H}}(0)\right) .
$$

We compare the two residuals, $r(x)$ and $r_{\mathrm{c}}(x)$, with the leading-order theoretical prediction $\mathrm{Da} f(x-$ k) $C_{\mathrm{H}}(x)$ from (4.4) in Figure 3 for a range of $(\mathrm{Pe}, \mathrm{Da})$-values. All quantities were scaled by $\left[\mathrm{Da} C_{\mathrm{H}}(0)\right]^{-1}$ to allow comparison across parameter values. For illustrative purposes, we plot the envelope of each function, indicating the fine-scale oscillatory behaviour via $f(x-k)$ in the inset to Figure 3(b). The present method works exceptionally well when uptake is weak relative to diffusion and advection (Figure $3 \mathrm{~g}, \mathrm{~h}$; the blue and pink regions overlap precisely) and deviates less than the classical method as uptake becomes more significant (Figure 3a,b,d,e; the blue region overlaps the pink region better than 


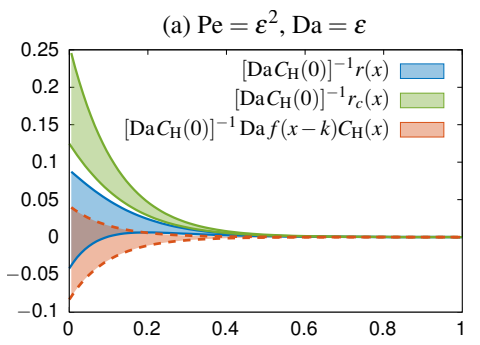

(b) $\mathrm{Pe}=\varepsilon, \mathrm{Da}=\varepsilon$

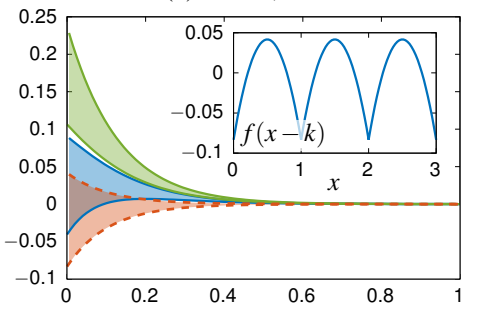

(e) $\mathrm{Pe}=\varepsilon, \mathrm{Da}=\varepsilon^{2}$
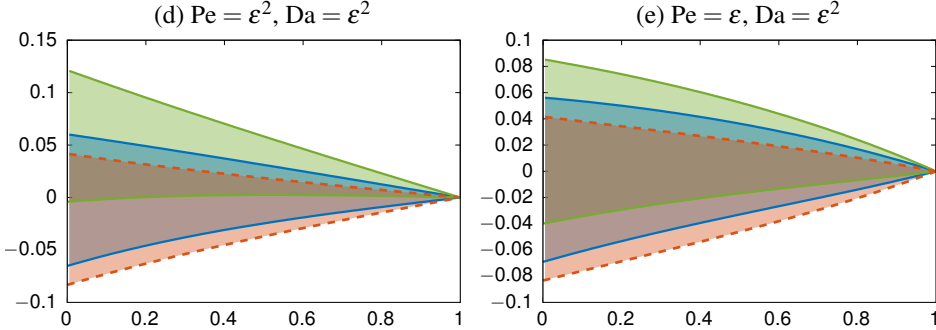

(h) $\mathrm{Pe}=\varepsilon, \mathrm{Da}=\varepsilon^{3}$
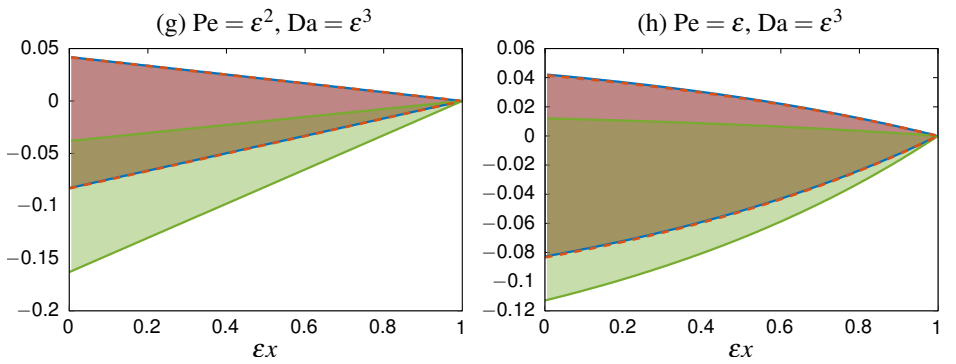

(c) $\mathrm{Pe}=1, \mathrm{Da}=\varepsilon$
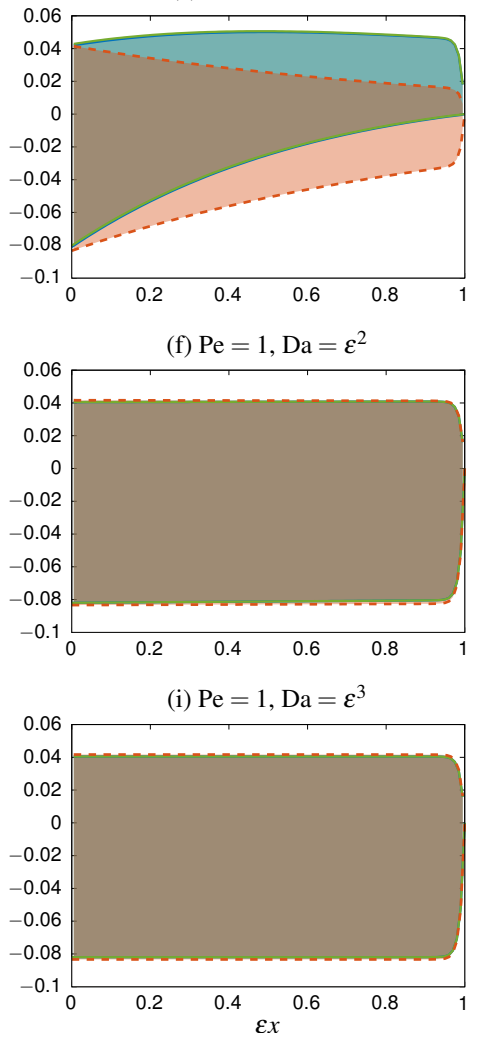

FIG. 3. Plots of envelopes of the residuals $\left[\mathrm{Da} C_{\mathrm{H}}(0)\right]^{-1} r(x)$ and $\left[\mathrm{Da} C_{\mathrm{H}}(0)\right]^{-1} r_{c}(x)$, each computed from a numerical solution of (2.4) for $C(x)$, compared with theoretical prediction of the leading-order oscillatory component $\left[\mathrm{Da} C_{\mathrm{H}}(0)\right]^{-1} \mathrm{Da} f(x-k) C_{\mathrm{H}}(x)$. We have normalised the data in each panel by the magnitude of the leading-order term in (4.4). There are $N=99$ sinks in all cases, so $\varepsilon=10^{-2}$; the $(\mathrm{Pe}, \mathrm{Da})$ values are as in Figure 2 and are indicated in the panels. The fine-scale oscillatory structure of residuals is shown in the inset to (b). The accuracy of the classical [new] method is illustrated by the degree of overlap between green [blue] and pink regions (the latter has a dashed boundary).

the green region does). Both methods share the same error when both advection and diffusion become strong, however (Figure 3c). Unlike the classical analysis, the present method does not assume unit-cell periodicity, which is perhaps where this contribution is lost. As Pavliotis \& Stuart (2008) point out in regard to the unit-cell problem, 'the local problem cannot really see the boundary — this is the key property of scale separation;' the present global method avoids this difficulty and is adaptable in principle to parameter ranges for which $G$ and $C_{\mathrm{H}}$ need not be (piecewise) slowly varying on the macroscale.

\section{Stochastic contributions}

\subsection{Normally perturbed sink locations}

We now consider sinks which are weakly perturbed from a periodic arrangement by normally-distributed random variables so that $\xi_{j}=j+\sigma \hat{\xi}_{j}$, where $\sigma \ll 1$ and $\hat{\xi}_{j} \sim \mathscr{N}(0,1)$. We assume that sinks do not change places as a result of the random perturbations. Using (3.12) and Taylor expanding around the 
12 of 28

periodic configuration,

$$
\hat{C}_{b}(x)=\operatorname{Da}\left\{\sum_{j \neq k}\left[\left.\sigma \hat{\xi}_{j}\left(G C_{\mathrm{H}}\right)_{y}\right|_{x, j}+\left.\frac{1}{2} \sigma^{2} \hat{\xi}_{j}^{2}\left(G C_{\mathrm{H}}\right)_{y y}\right|_{x, j}+\cdots\right]+\left[\left(G C_{\mathrm{H}}\right)\right]_{y=k}^{y=k+\sigma \hat{\xi}_{k}}\right\},
$$

where the cell in which the coordinate $x$ falls, $k=\left\lfloor x+\frac{1}{2}\right\rfloor$, is again treated separately to avoid expanding non-smooth functions. The contribution from $O\left(\varepsilon^{-1}\right)$ terms in the sum will be an order of magnitude greater than that from the single unit cell $k$ as $\varepsilon \rightarrow 0$, which we therefore neglect. Assuming that the sinks are independently distributed, which implies $\operatorname{Cov}\left(\hat{\xi}_{j}, \hat{\xi}_{\ell}\right)=\delta_{j, \ell}$, we write the covariance as

$$
\begin{aligned}
\operatorname{Cov}\left(\hat{C}_{b}\left(x_{1}\right), \hat{C}_{b}\left(x_{2}\right)\right) & =\left.\left.\mathrm{Da}^{2} \sigma^{2} \sum_{j \neq k_{1}} \sum_{\ell \neq k_{2}}\left(G C_{\mathrm{H}}\right)_{y}\right|_{x_{1}, j}\left(G C_{\mathrm{H}}\right)_{y}\right|_{x_{2}, \ell} \operatorname{Cov}\left(\hat{\xi}_{j}, \hat{\xi}_{\ell}\right)+\cdots \\
& =\left.\left.\mathrm{Da}^{2} \sigma^{2} \sum_{j \notin\left\{k_{1}, k_{2}\right\}}\left(G C_{\mathrm{H}}\right)_{y}\right|_{x_{1}, j}\left(G C_{\mathrm{H}}\right)_{y}\right|_{x_{2}, j}+\cdots
\end{aligned}
$$

where $k_{i} \equiv\left\lfloor x_{i}+\frac{1}{2}\right\rfloor$, for $i=1,2$. Using (D.4) to approximate sums with integrals,

$$
\operatorname{Cov}\left(\hat{C}_{b}\left(x_{1}\right), \hat{C}_{b}\left(x_{2}\right)\right)=\left.\left.\mathrm{Da}^{2} \sigma^{2} \int_{0}^{\mathcal{E}^{-1}}\left(G C_{\mathrm{H}}\right)_{y}\right|_{x_{1}, y}\left(G C_{\mathrm{H}}\right)_{y}\right|_{x_{2}, y} \mathrm{~d} y+\cdots
$$

As observed previously in related problems (Chernyavsky et al., 2011, 2012; Heitzinger \& Ringhofer, 2014; Russell et al., 2016) the fluctuations at a given location depend non-locally on the concentration profile throughout the domain, despite the small and independent perturbations to the sink locations.

In Figure 4 the theoretical prediction of the variance, $\operatorname{Var}(C(x)) \equiv \operatorname{Cov}\left(\hat{C}_{b}(x), \hat{C}_{b}(x)\right)$, and transverse covariance, $\operatorname{Cov}^{\mathrm{T}}(C(x)) \equiv \operatorname{Cov}\left(\hat{C}_{b}(x), \hat{C}_{b}\left(\varepsilon^{-1}-x\right)\right)$, given by (5.2) are compared with the sample statistics of an ensemble of $10^{4}$ Monte-Carlo simulations of the transport equation (2.4), computed using the method described in Appendix A, with sink locations drawn from a normal distribution with variance $\sigma^{2}=10^{-2}$. The agreement is excellent for $\mathrm{Pe}=O(\varepsilon)$ or smaller (Figure 4a,b,d,e,g,h). When advection is stronger, with $\mathrm{Pe}=O(1)$, sink-to-sink oscillations emerge in the Monte-Carlo estimates of $\operatorname{Var}(C)$. Nevertheless the approximation (5.2) captures its slowly varying mean value, and its lack of correlation across the domain (reflected by a spike in $\operatorname{Cov}^{\mathrm{T}}(C)$ ); we expect higher-order terms to describe the oscillating part of the variance.

Taking the average of (5.1) across realisations of sink distributions gives

$$
\left\langle\hat{C}_{b}(x)\right\rangle=\mathrm{Da}\left\{\sum_{j \neq k}\left[\left.\frac{1}{2} \sigma^{2}\left(G C_{\mathrm{H}}\right)_{y y}\right|_{x, j}+\cdots\right]+\left\langle\left[\left(G C_{\mathrm{H}}\right)\right]_{y=k}^{y=k+\sigma \hat{\xi}_{k}}\right\rangle\right\} .
$$

Again neglecting the small cell- $k$ term and approximating sums with integrals we find

$$
\begin{aligned}
\left\langle\hat{C}_{b}(x)\right\rangle & =\left.\frac{1}{2} \operatorname{Da}^{2} \int_{0}^{\varepsilon^{-1}}\left(G C_{\mathrm{H}}\right)_{y y}\right|_{x, y} \mathrm{~d} y+\cdots \\
& =\frac{1}{2} \operatorname{Da} \sigma^{2}\left[\left.\left(G C_{\mathrm{H}}\right)_{y}\right|_{x, \varepsilon^{-1}}-\left.\left(G C_{\mathrm{H}}\right)_{y}\right|_{x, x^{+}}+\left.\left(G C_{\mathrm{H}}\right)_{y}\right|_{x, x^{-}}-\left.\left(G C_{\mathrm{H}}\right)_{y}\right|_{x, 0}\right]+\cdots \\
& =\frac{1}{2} \operatorname{Da} \sigma^{2}\left[-C_{\mathrm{H}}(x)-\left.\left(G C_{\mathrm{H} y}\right)\right|_{x, 0}\right]+\cdots
\end{aligned}
$$

where we have applied the jump condition (A.1) and used $\left.G_{y}\right|_{x, 0}=0$. 
(a) $\mathrm{Pe}=\varepsilon^{2}, \mathrm{Da}=\varepsilon$

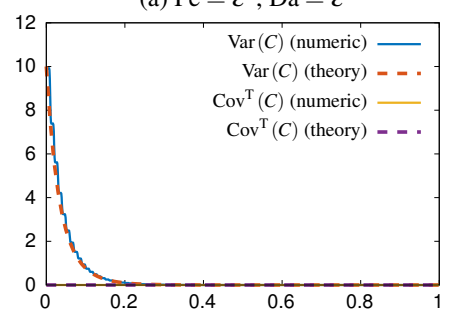

(d) $\mathrm{Pe}=\varepsilon^{2}, \mathrm{Da}=\varepsilon^{2}$

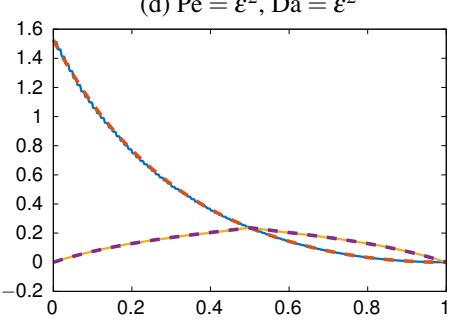

(g) $\mathrm{Pe}=\varepsilon^{2}, \mathrm{Da}=\varepsilon^{3}$

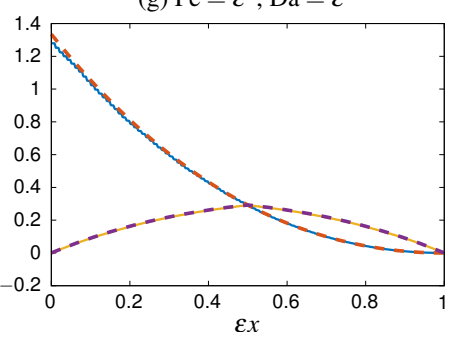

(b) $\mathrm{Pe}=\varepsilon, \mathrm{Da}=\varepsilon$

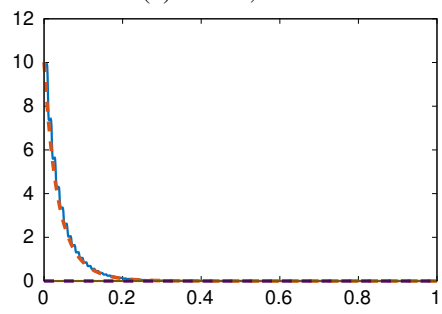

(e) $\mathrm{Pe}=\varepsilon, \mathrm{Da}=\varepsilon^{2}$

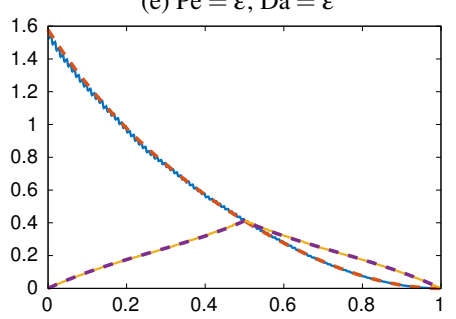

(h) $\mathrm{Pe}=\varepsilon, \mathrm{Da}=\varepsilon^{3}$

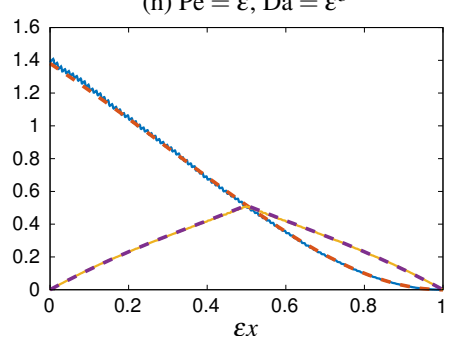

(c) $\mathrm{Pe}=1, \mathrm{Da}=\varepsilon$

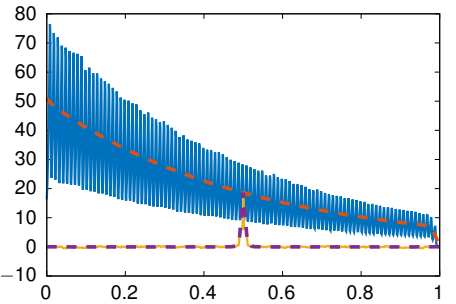

(f) $\mathrm{Pe}=1, \mathrm{Da}=\varepsilon^{2}$

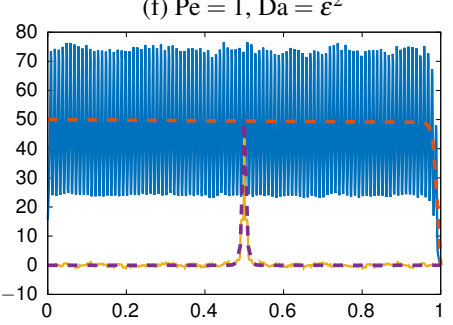

(i) $\mathrm{Pe}=1, \mathrm{Da}=\varepsilon^{3}$

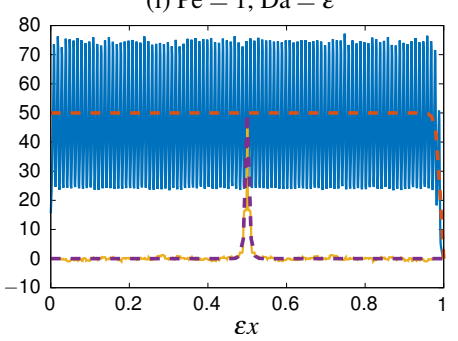

FIG. 4. Comparison between the sample variance (solid blue) and transverse covariance (solid orange) generated from $10^{4}$ solutions of (2.4) with normally perturbed sink locations, $\xi_{j}=j+\sigma \mathscr{N}(0,1)$, with $\sigma=0.1$, and the theoretical prediction (5.2) of variance (dashed red) and transverse covariance (dashed purple). All variances and transverse covariances have been scaled by $\varepsilon^{-1}\left[\mathrm{Da} \sigma C_{\mathrm{H}}(0) G(0,0)\right]^{-2}$. Other parameters are as in Figure 2.

Using the scaling strategy outlined earlier to analyse $(5.2,5.4)$ in the distinguished limit, we see that the dominant contribution to the fluctuating part of $\hat{C}_{b}$ has magnitude $\varepsilon^{3 / 2} \sigma$ and its mean part has magnitude $\varepsilon^{2} \sigma^{2}$. We therefore expect the correction to $\langle C\rangle$ due to stochasticity to be sub-dominant to $\hat{C}_{a}$ provided $\sigma \ll 1$. Figure 5 compares the mean residual,

$$
\left\langle r_{n}(x)\right\rangle=\langle r(x)\rangle+\frac{1}{2} \mathrm{Da}^{2}\left[C_{\mathrm{H}}(x)+\left.\left(G C_{\mathrm{H} y}\right)\right|_{x, 0}\right]
$$

(where (4.7) is modified using (5.4)), with the leading-order oscillatory term, $\operatorname{Da} f(x-k) C_{\mathrm{H}}(x)$. The magnitude and overall shape of the mean residuals are similar to those from the periodic array (see Figure 3), although with a slightly reduced amplitude in some cases. This similarity demonstrates the sub-dominant effect of the weak stochasticity on the mean compared with the periodic corrections in this instance. Also shown in Figure 5 is the prediction of how the lower envelope of the oscillatory mean residual is elevated as a result of averaging (see Appendix E): averaging an ensemble of 'spiky' oscillations of the kind shown in Figure 3(b), with each member of the ensemble displaced laterally by a small normally distributed distance, leads to a smoother mean waveform with an elevated minimum. 
(a) $\mathrm{Pe}=\varepsilon^{2}, \mathrm{Da}=\varepsilon$

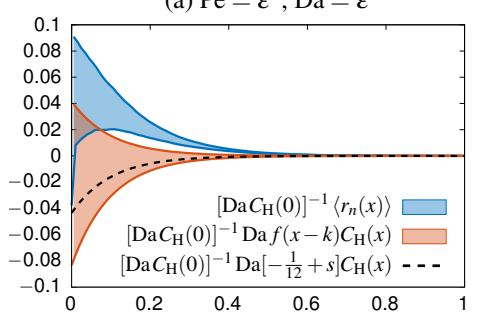

(d) $\mathrm{Pe}=\varepsilon^{2}, \mathrm{Da}=\varepsilon^{2}$

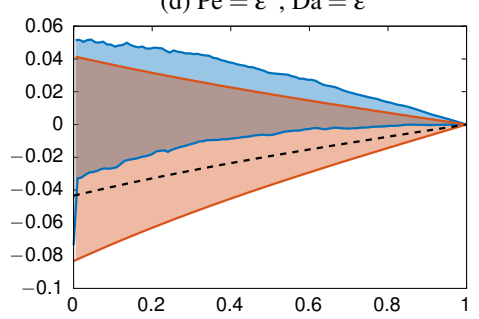

(g) $\mathrm{Pe}=\varepsilon^{2}, \mathrm{Da}=\varepsilon^{3}$

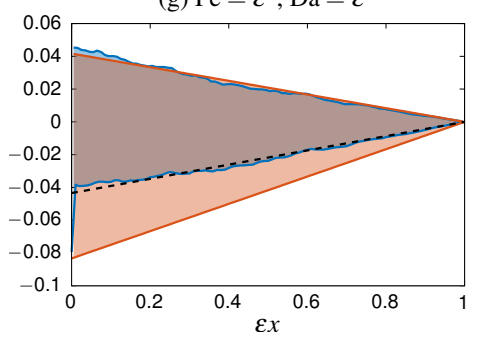

(b) $\mathrm{Pe}=\varepsilon, \mathrm{Da}=\varepsilon$

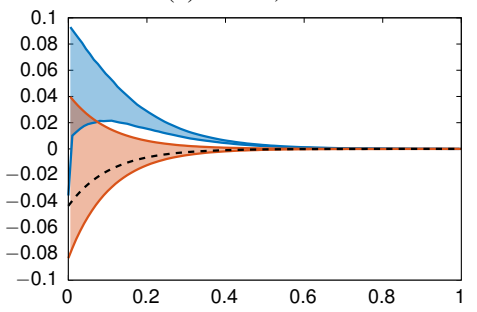

(e) $\mathrm{Pe}=\varepsilon, \mathrm{Da}=\varepsilon^{2}$

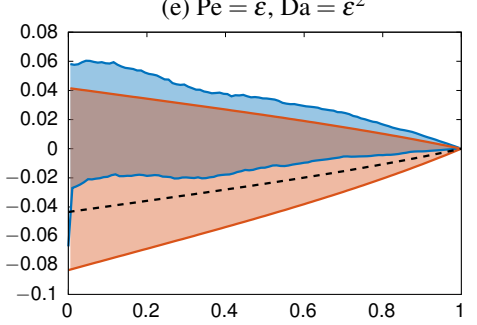

(h) $\mathrm{Pe}=\varepsilon, \mathrm{Da}=\varepsilon^{3}$

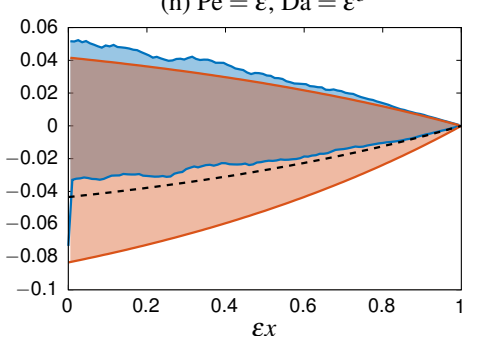

(c) $\mathrm{Pe}=1, \mathrm{Da}=\varepsilon$

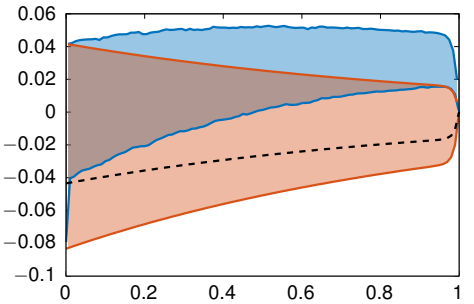

(f) $\mathrm{Pe}=1, \mathrm{Da}=\varepsilon^{2}$

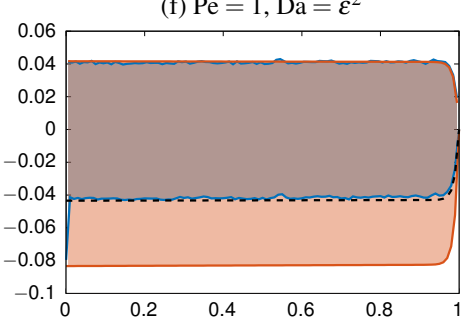

(i) $\mathrm{Pe}=1, \mathrm{Da}=\varepsilon^{3}$

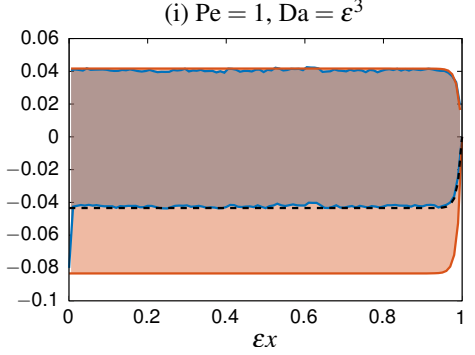

FIG. 5. Plots of $\left[\mathrm{Da} C_{\mathrm{H}}(0)\right]^{-1}\left\langle r_{n}(x)\right\rangle$, computed from $10^{4}$ numerical solutions of (2.4) for $C(x)$ with normally perturbed sink locations, $\xi_{j}=j+\sigma \mathscr{N}(0,1)$, with $\sigma=0.1$, compared with $\left[\mathrm{Da} C_{\mathrm{H}}(0)\right]^{-1} \mathrm{Da} f(x-k) C_{\mathrm{H}}(x)$. Quantities are normalised in the same way as in Figure 3. Other parameters are as in Figure 2. The pink regions match those in Figure 3; dashed, black lines show the impact of averaging on their lower envelope, derived in Appendix E, where $s=\sigma / \sqrt{2 \pi}$. The blue regions incorporate the correction $\left\langle\hat{C}_{b}\right\rangle$. The accuracy of the approximation is illustrated by the degree of overlap between blue region and the pink region above the dashed line.

This refined lower boundary agrees very well with simulations for small Da, but deviations develop as $\mathrm{Da}$ is increased.

Finally, we may use $\left.\left(\hat{C}_{b}\right)_{x}\right|_{\mathcal{E}^{-1}}$ (see (2.5)) to estimate the uncertainty in the total solute uptake by sinks as

$$
\operatorname{Var}\left(\left.\left(\hat{C}_{b}\right)_{x}\right|_{\mathcal{E}^{-1}}\right)=\left.\operatorname{Da}^{2} \sigma^{2} \int_{0}^{\varepsilon^{-1}}\left(G_{x} C_{\mathrm{H}}\right)_{y}\right|_{\mathcal{E}^{-1}, y} \mathrm{~d} y .
$$

\subsection{Uniformly random sink locations}

We now consider an array of $N$ point sinks, the locations of which are drawn from a uniform distribution $\mathscr{U}\left(0, \varepsilon^{-1}\right)$, where $\varepsilon=1 /(N+1)$, and sorted into ascending order: $\xi_{1} \leqslant \xi_{2} \leqslant \cdots \leqslant \xi_{N}$. Thus $\xi_{j}$ is the 
$j$-th order statistic from a set of $N$ uniform random variables (Ahsanullah et al., 2013). In contrast to the array of weakly perturbed sinks, we cannot Taylor expand around the periodic configuration and neglect the single unit cell which contains the coordinate $x$. This is because the strong disorder allows sinks to leave their unit cells and change order. Instead, we use results on order statistics to approximate the moments of the concentration profile.

We first note that the sum over all $N$ order statistics $\xi_{j}$ is equal to the sum of the $N$ underlying uniform random variables; the former is just a permutation of the latter. This basic fact is used in David \& Nagaraja (2003) and Chunsheng (1992) to prove identities involving sums of moments of order statistics. In particular, we will use the following identity,

$$
\sum_{j \neq k} \operatorname{Cov}\left(g\left(X_{j: N}\right), h\left(X_{k: N}\right)\right)=\sum_{j=1}^{N}\left[\left\langle g\left(X_{j: N}\right)\right\rangle\left\langle h\left(X_{j: N}\right)\right\rangle-\langle g(X)\rangle\langle h(X)\rangle\right],
$$

where $X$ is a random variable (with finite variance), $X_{j: N}$ denotes the $j$-th order statistic out of a sample of size $N$ from the distribution of $X$, and $g, h$ are any real-valued functions satisfying $\operatorname{Var}(g(X))<\infty$, $\operatorname{Var}(h(X))<\infty$.

Let the uniform random variable $U \sim \mathscr{U}\left(0, \varepsilon^{-1}\right)$ with probability density function $f_{U}(x)=\varepsilon, 0 \leqslant$ $x \leqslant \varepsilon^{-1}$. Using (3.12), we write the covariance as

$$
\begin{aligned}
\operatorname{Cov}\left(\hat{C}_{b}\left(x_{1}\right), \hat{C}_{b}\left(x_{2}\right)\right)= & \operatorname{Da}^{2} \sum_{j=1}^{N} \sum_{k=1}^{N} \operatorname{Cov}\left(\left(G C_{\mathrm{H}}\right)_{x_{1}, \xi_{j}},\left(G C_{\mathrm{H}}\right)_{x_{2}, \xi_{k}}\right) \\
= & \operatorname{Da}^{2}\left[\sum_{j \neq k} \operatorname{Cov}\left(\left(G C_{\mathrm{H}}\right)_{x_{1}, \xi_{j}},\left(G C_{\mathrm{H}}\right)_{x_{2}, \xi_{k}}\right)+\sum_{j=1}^{N} \operatorname{Cov}\left(\left(G C_{\mathrm{H}}\right)_{x_{1}, \xi_{j}},\left(G C_{\mathrm{H}}\right)_{x_{2}, \xi_{j}}\right)\right] \\
= & \operatorname{Da}^{2}\left\{\sum_{j=1}^{N}\left\langle\left.\left(G C_{\mathrm{H}}\right)\right|_{x_{1}, \xi_{j}}\right\rangle\left\langle\left.\left(G C_{\mathrm{H}}\right)\right|_{x_{2}, \xi_{j}}\right\rangle-N\left\langle\left.\left(G C_{\mathrm{H}}\right)\right|_{x_{1}, U}\right\rangle\left\langle\left.\left(G C_{\mathrm{H}}\right)\right|_{x_{2}, U}\right\rangle\right. \\
& \left.\quad+\sum_{j=1}^{N}\left[\left\langle\left.\left.\left(G C_{\mathrm{H}}\right)\right|_{x_{1}, \xi_{j}}\left(G C_{\mathrm{H}}\right)\right|_{x_{2}, \xi_{j}}\right\rangle-\left\langle\left.\left(G C_{\mathrm{H}}\right)\right|_{x_{1}, \xi_{j}}\right\rangle\left\langle\left.\left(G C_{\mathrm{H}}\right)\right|_{x_{2}, \xi_{j}}\right\rangle\right]\right\} \\
= & N \operatorname{Da}^{2}\left[\left\langle\left.\left.\left(G C_{\mathrm{H}}\right)\right|_{x_{1}, U}\left(G C_{\mathrm{H}}\right)\right|_{x_{2}, U}\right\rangle-\left\langle\left.\left(G C_{\mathrm{H}}\right)\right|_{x_{1}, U}\right\rangle\left\langle\left.\left(G C_{\mathrm{H}}\right)\right|_{x_{2}, U}\right\rangle\right],
\end{aligned}
$$

where we have used (5.7) to transform averages over the order statistics into averages over the uniform variable $U$. Writing the averages as integrals using the probability density function $f_{U}$ then yields

$$
\begin{aligned}
& \operatorname{Cov}\left(\hat{C}_{b}\left(x_{1}\right), \hat{C}_{b}\left(x_{2}\right)\right)= \\
& (1-\varepsilon) \operatorname{Da}^{2}\left[\left.\left.\int_{0}^{\varepsilon^{-1}}\left(G C_{\mathrm{H}}\right)\right|_{x_{1}, y}\left(G C_{\mathrm{H}}\right)\right|_{x_{2}, y} \mathrm{~d} y-\left.\left.\varepsilon \int_{0}^{\varepsilon^{-1}}\left(G C_{\mathrm{H}}\right)\right|_{x_{1}, y} \mathrm{~d} y \int_{0}^{\varepsilon^{-1}}\left(G C_{\mathrm{H}}\right)\right|_{x_{2}, y} \mathrm{~d} y\right] .
\end{aligned}
$$

We have made no further approximations to arrive at this expression for the covariance of $\hat{C}_{b}$ and it contains contributions at different orders of $\varepsilon$. A leading-order estimate is obtained by retaining only the 1 in $(1-\varepsilon)$, from which we find that the covariance has magnitude $\varepsilon$ in the distinguished limit, implying fluctuations around the mean have magnitude $\varepsilon^{1 / 2}$.

In Figure 6 we compare the theoretical prediction of the variance and transverse covariance, in (5.9), with sample statistics of an ensemble of $10^{4}$ Monte-Carlo simulations of (2.4), with sink locations drawn 

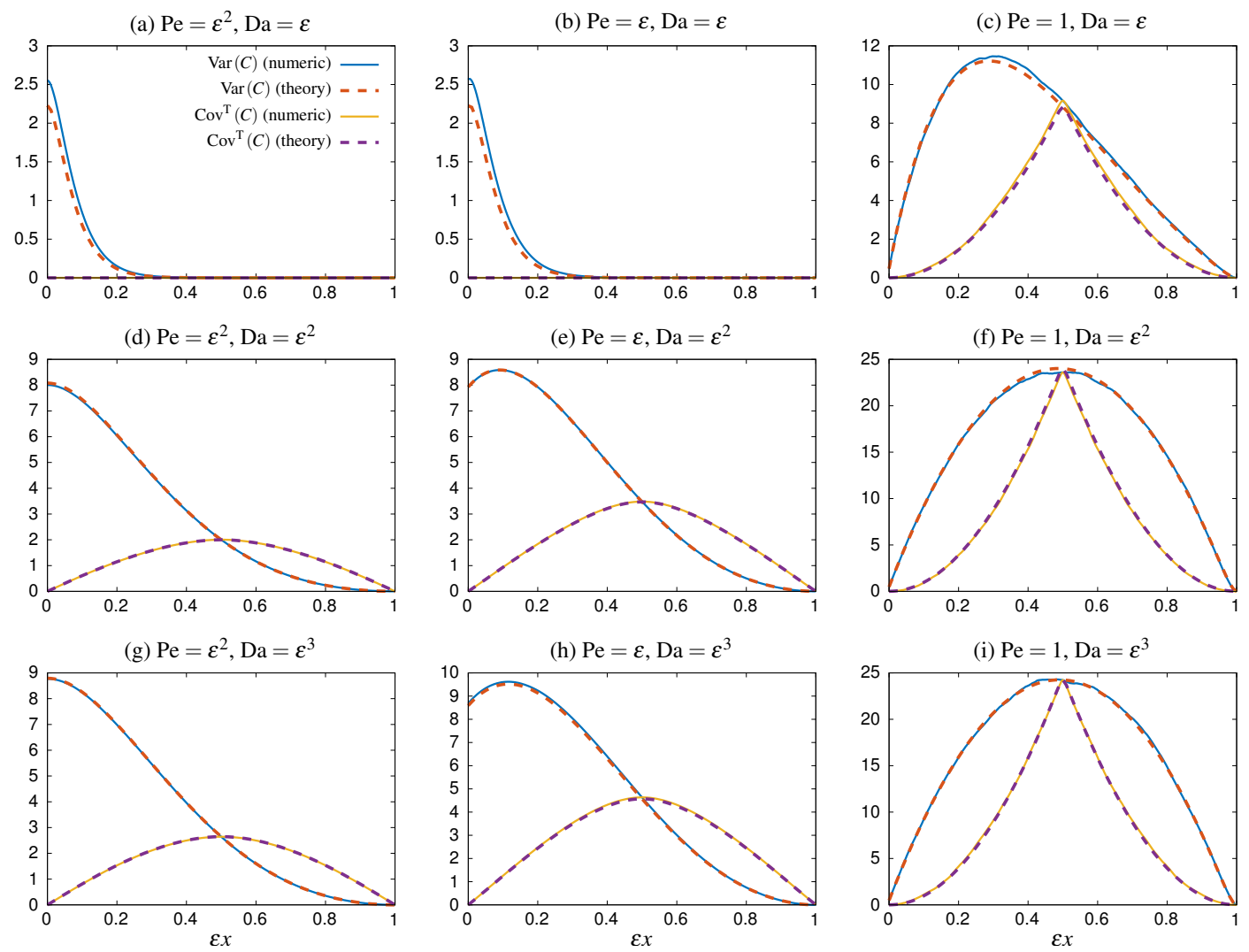

FIG. 6. Comparison between the sample variance (solid blue) and transverse covariance (solid orange) generated from $10^{4}$ solutions of (2.4) with the $\xi_{j}$ drawn from $\mathscr{U}\left(0, \varepsilon^{-1}\right)$ and then sorted into ascending order for each realisation, and the theoretical prediction (5.9). All variances and transverse covariances have been scaled by $\left[\mathrm{Da} C_{\mathrm{H}}(0) G(0,0)\right]^{-2}$. Other parameters are as in Figure 2.

from a uniform distribution $\mathscr{U}\left(0, \varepsilon^{-1}\right)$ and labelled in ascending order. For all values of $(\mathrm{Pe}, \mathrm{Da})$ shown, the agreement is excellent, except perhaps for a small discrepancy near the inlet when $\mathrm{Da}=O(\varepsilon)$ and $\mathrm{Pe} \ll 1$. In contrast to Figure 4, sink-to-sink oscillations in the variance or transverse covariance are not visible here. We expect that oscillations appear at higher order and are sub-dominant to the effects of strongly disordered sink locations.

The variance in the net uptake by sinks, from (2.5), is derived analogously to (5.9) as

$$
\operatorname{Var}\left(\left.\left(\hat{C}_{b}\right)_{x}\right|_{\mathcal{E}^{-1}}\right)=\mathrm{Da}^{2}\left[\int_{0}^{\varepsilon^{-1}}\left[\left.\left(G_{x} C_{\mathrm{H}}\right)\right|_{\mathcal{E}^{-1}, y}\right]^{2} \mathrm{~d} y-\varepsilon\left[\left.\int_{0}^{\varepsilon^{-1}}\left(G_{x} C_{\mathrm{H}}\right)\right|_{\mathcal{E}^{-1}, y} \mathrm{~d} y\right]^{2}\right]
$$

Interestingly, simulations show that $\left.\left(\hat{C}_{b}\right)_{x}\right|_{\varepsilon^{-1}}$ has a roughly Gaussian distribution for $\mathrm{Pe}=O(\varepsilon)$ and $\mathrm{Da}=O\left(\varepsilon^{2}\right)$, but an asymmetric distribution when $\mathrm{Pe}=O(1)$ (not shown).

We now turn to the mean of the first stochastic correction. Using again the equivalence of sums over 
order statistics and their underlying random variables, the mean of $\hat{C}_{b}$ can be written as

$$
\begin{aligned}
\left\langle\hat{C}_{b}(x)\right\rangle & =\mathrm{Da} \sum_{j=1}^{N}\left[\left\langle\left(G C_{\mathrm{H}}\right)_{x, \xi_{j}}\right\rangle-\left.\left(G C_{\mathrm{H}}\right)\right|_{x, j}\right] \\
& =\mathrm{Da} \sum_{j=1}^{N}\left[\left\langle\left(G C_{\mathrm{H}}\right)_{x, U}\right\rangle-\left.\left(G C_{\mathrm{H}}\right)\right|_{x, j}\right] \\
& =\mathrm{Da}\left[\left.(1-\varepsilon) \int_{0}^{\varepsilon^{-1}}\left(G C_{\mathrm{H}}\right)\right|_{x, y} \mathrm{~d} y-\left.\sum_{j=1}^{N}\left(G C_{\mathrm{H}}\right)\right|_{x, j}\right] .
\end{aligned}
$$

In the distinguished limit, the first integral in the brackets is $O\left(\varepsilon^{-2}\right)$, the second integral is $O\left(\varepsilon^{-1}\right)$, and the sum is $O\left(\varepsilon^{-2}\right)$. Converting the sum to an integral, we expect the $O\left(\varepsilon^{-2}\right)$ contributions to cancel the first integral, so we retain terms up to the size of the smaller integral term,

$$
\begin{aligned}
\left.\sum_{j=1}^{N}\left(G C_{\mathrm{H}}\right)\right|_{x, j} & =\left.\int_{0}^{\varepsilon^{-1}}\left(G C_{\mathrm{H}}\right)\right|_{x, y} \mathrm{~d} y-\frac{1}{2}\left[\left.\left(G C_{\mathrm{H}}\right)\right|_{x, 0}+\left.\left(G C_{\mathrm{H}}\right)\right|_{x, \varepsilon^{-1}}\right]+\cdots \\
& =\left.\int_{0}^{\varepsilon^{-1}}\left(G C_{\mathrm{H}}\right)\right|_{x, y} \mathrm{~d} y+\frac{1}{2} \varepsilon^{-1} C_{\mathrm{H}}(0) C_{\mathrm{H}}(x)+\cdots,
\end{aligned}
$$

where we have applied the outlet boundary condition (2.4b) and (3.6). Using this approximation, we find

$$
\left\langle\hat{C}_{b}(x)\right\rangle=\mathrm{Da}\left[-\left.\varepsilon \int_{0}^{\varepsilon^{-1}}\left(G C_{\mathrm{H}}\right)\right|_{x, y} \mathrm{~d} y-\frac{1}{2} \varepsilon^{-1} C_{\mathrm{H}}(0) C_{\mathrm{H}}(x)+O(1)\right],
$$

which has magnitude $O(\varepsilon)$ in the distinguished limit.

Figure 7 compares the mean residual $\langle r(x)\rangle$, calculated using (4.7) from $10^{5}$ Monte-Carlo samples, each with sink locations drawn from a uniform distribution, and the theoretical prediction (5.13). We compare the sample statistics with this $O(\varepsilon)$ stochastic contribution rather than the $O\left(\varepsilon^{2}\right)$ oscillating part, $\operatorname{Da} f(x-k) C_{\mathrm{H}}(x)$, since the sink-to-sink oscillations appear at higher order here. The theory predicts the overall magnitude and shape of the correction to the mean concentration for many parameter values, especially when $\mathrm{Da}$ is small, and when Pe is large. However, some features, such as near the inlet boundary in Figure $7(a, b)$ are not captured by the leading-order theory for this choice of $\varepsilon$; we expect that further correction terms will account for these discrepancies.

\subsection{Magnitude estimates in other regions of parameter space}

Following Russell et al. (2016), we can identify three parameter regimes around the distinguished limit. In each regime, either diffusion [D], advection [A] or uptake [U] is the dominant process. These can be identified by balancing the various terms in (3.1). [D] is defined by the region $\mathrm{Pe} \ll \varepsilon, \mathrm{Da} \ll \varepsilon^{2}$; [A] by $\mathrm{Pe} \gg \varepsilon, \mathrm{Da} \ll \mathrm{Pe}^{2}$; and [U] by $\mathrm{Da} \gg \max \left(\varepsilon^{2}, \mathrm{Pe}^{2}\right)$. In [D], $C_{\mathrm{H}}$ varies smoothly across the whole domain over a lengthscale $x \sim \varepsilon^{-1}$ (see Figure 2(g)). In [U], the lengthscale shrinks to $x \sim \mathrm{Da}^{-1 / 2}$ (Figure 2a,b). In [A], for $\varepsilon \mathrm{Pe} \ll \mathrm{Da} \ll \mathrm{Pe}^{2}$ this lengthscale increases to $x \sim \mathrm{Pe} / \mathrm{Da}$ (we denote this subregion $\left[\mathrm{A}_{I}\right]$ ), and then encompasses the whole domain for $\mathrm{Da} \ll \varepsilon \mathrm{Pe}$ (with a short boundary layer of length $x \sim 1 / \mathrm{Pe}$ at the outlet; this is subregion $\left[\mathrm{A}_{I I}\right]$, see Figure 2(f,i)). Other panels in Figure 2 sit at interfaces between these regions: (c) $\left[\mathrm{A}_{I} / \mathrm{A}_{I I}\right]$; (d) $[\mathrm{D} / \mathrm{U}]$; (e) $[\mathrm{A} / \mathrm{D} / \mathrm{U}]$, the central distinguished limit; and (h) $[\mathrm{D} / \mathrm{A}]$. We 

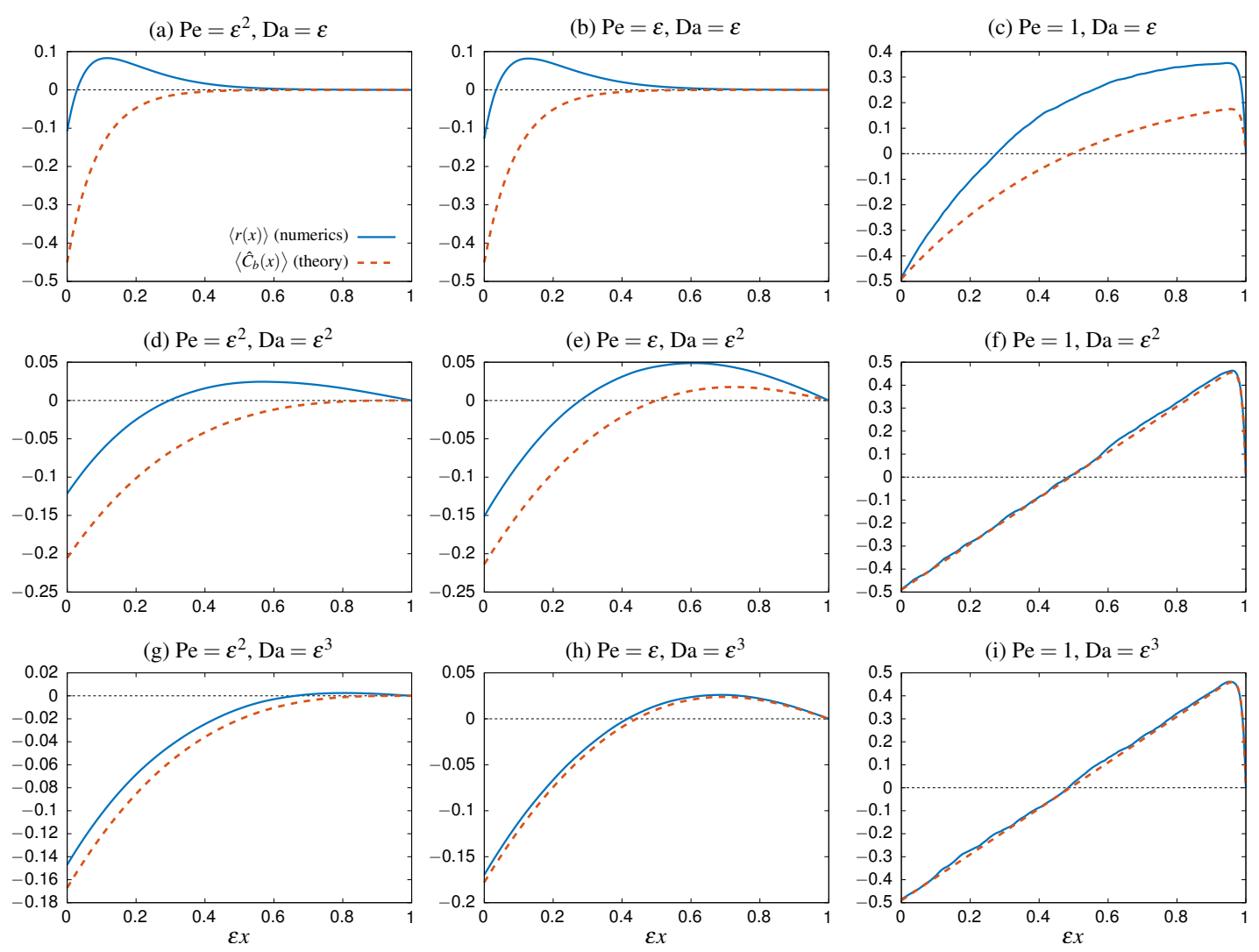

FIG. 7. Plots of $\langle r(x)\rangle$, each computed from $10^{4}$ numerical solutions of (2.4) for $C(x)$ with uniformly distributed sinks, $\xi_{j}$ drawn from $\mathscr{U}\left(0, \varepsilon^{-1}\right)$, and the theoretical prediction (5.13). All quantities have been scaled by $\varepsilon \mathrm{Da}^{-1} C_{\mathrm{H}}(0)^{-2}$. Other parameters are as in Figure 2.

use these lengthscales to estimate the asymptotic magnitudes of $C_{\mathrm{H}}$ and $G$ in these regions of parameter space, from which we deduce the magnitudes of the deterministic correction $\hat{C}_{a}, \hat{C}_{a a}$ in terms of $C_{\mathrm{H}}$ and $G$. These results are summarised in Table 1 .

The macroscale approximation is slowly varying when $\mathrm{Pe} \ll 1$ (for the downstream boundary layer to extend over multiple sinks) and $\mathrm{Da} \ll 1$ (ensuring the corrections due to discreteness to remain subdominant to $C_{\mathrm{H}}$ ). Oscillatory corrections in $\hat{C}_{a}$ grow as each of these boundaries in parameter space is approached, indicating how $C_{\mathrm{H}}$ becomes an increasingly poor approximation of the gradient of the exact solution over short lengthscales.

Turning to the stochastic corrections, we estimate the asymptotic magnitudes of the mean and fluctuations (given by the standard deviation) of $\hat{C}_{b}$ for both normally perturbed and uniformly-random sink locations. These magnitudes are summarised in Table 2. It turns out that the domain of validity of $C_{\mathrm{H}}$ remains $\mathrm{Da} \ll 1$ and $\mathrm{Pe} \ll 1$ in all cases. However in the uniformly-random case, the dominant correction to $C_{\mathrm{H}}$ is due to randomness, whereas for normally perturbed sinks, the discrete correction dominates the stochastic one. 


\begin{tabular}{c|cccc} 
Region & $C_{\mathrm{H}}$ & $\mathrm{G}$ & $\hat{C}_{a}$ & $\hat{C}_{a a}$ \\
\hline $\mathrm{A} / \mathrm{D} / \mathrm{U}$ & 1 & $\varepsilon^{-1}$ & $\varepsilon(1, \varepsilon)$ & $\varepsilon^{2}$ \\
$\mathrm{D}$ & 1 & $\varepsilon^{-1}$ & $\mathrm{Da}^{-1}(1, \varepsilon)$ & $\mathrm{Da}^{2} \varepsilon^{-2}$ \\
$\mathrm{U}$ & $\varepsilon \mathrm{Da}^{-1 / 2}$ & $\mathrm{Da}^{-1 / 2}$ & $\varepsilon\left(1, \mathrm{Da}^{1 / 2}\right)$ & $\varepsilon \mathrm{Da}^{1 / 2}$ \\
$\mathrm{~A}$ & $\varepsilon \mathrm{Pe}^{-1}$ & $\mathrm{Pe}^{-1}$ & $\varepsilon \mathrm{DaPe}^{-2}(1, \mathrm{Pe})$ & $\varepsilon \mathrm{Da}^{2} \mathrm{Pe}^{-3}$
\end{tabular}

Table 1. Asymptotic magnitudes of the leading-order homogenized solution $C_{\mathrm{H}}$ and the Green's function $G$ in various parameter regimes (defined in the text), with corresponding estimates of the magnitudes of the deterministic corrections $\hat{C}_{a}$ and $\hat{C}_{a a}$. In the $\hat{C}_{a}$ column, the first term in the parentheses corresponds to the leading-order slowly varying terms and the second to the amplitude of leading-order oscillatory terms.

\begin{tabular}{c|cc|cc} 
& \multicolumn{2}{|c|}{ Normally perturbed } & \multicolumn{2}{c}{ Uniform } \\
Region & $\left\langle\hat{C}_{b}\right\rangle$ & $\sqrt{\operatorname{Var}\left(\hat{C}_{b}\right)}$ & $\left\langle\hat{C}_{b}\right\rangle$ & $\sqrt{\operatorname{Var}\left(\hat{C}_{b}\right)}$ \\
\hline $\mathrm{A} / \mathrm{D} / \mathrm{U}$ & $\sigma^{2} \varepsilon^{2}$ & $\sigma \varepsilon^{3 / 2}$ & $\varepsilon$ & $\varepsilon^{1 / 2}$ \\
$\mathrm{D}$ & $\sigma^{2} \mathrm{Da}$ & $\sigma \varepsilon^{-1 / 2} \mathrm{Da}$ & $\varepsilon^{-1} \mathrm{Da}$ & $\varepsilon^{-3 / 2} \mathrm{Da}$ \\
$\mathrm{U}$ & $\sigma^{2} \varepsilon \mathrm{Da}^{1 / 2}$ & $\sigma \varepsilon \mathrm{Da}^{1 / 4}$ & $\varepsilon$ & $\varepsilon \mathrm{Da}^{-1 / 4}$ \\
$\mathrm{~A}_{I}$ & $\sigma^{2} \varepsilon \mathrm{DaPe}^{-1}$ & $\sigma \varepsilon^{3 / 2} \mathrm{DaPe}^{-2}$ & $\varepsilon \mathrm{DaPe}^{-2}$ & $\varepsilon^{1 / 2} \mathrm{DaPe}^{-2}$ \\
$\mathrm{~A}_{I I}$ & $\sigma^{2} \varepsilon \mathrm{DaPe}^{-1}$ & $\sigma \varepsilon \mathrm{Da}^{3 / 2} \mathrm{Pe}^{-5 / 2}$ & $\varepsilon \mathrm{DaPe}^{-2}$ & $\varepsilon \mathrm{Da}^{1 / 2} \mathrm{Pe}^{-3 / 2}$
\end{tabular}

Table 2. Magnitude estimates of the mean and fluctuations of the stochastic correction $\hat{C}_{b}$

\section{Discussion}

We have analysed a model of transport past an array of point sinks with first-order uptake kinetics. We considered periodic, weakly perturbed and strongly disordered arrays; weak disorder was modelled using small normally-distributed perturbations from a periodic configuration while strongly disordered sinks had uniformly-randomly distributed locations. We posed an ad hoc expansion for the concentration field, centred around the leading-order homogenized concentration (which is obtained using classical two-scale methods described in Appendix B), in which the higher-order terms can be identified as corrections due to the discrete nature of the sinks and the effects of disorder, and combinations thereof. However, the asymptotic ordering of the expansion is not known a priori and it contains a mixture of expressions varying on long and short lengthscales and having deterministic and stochastic components. We assessed the magnitudes of the different correction terms in the distinguished limit $\mathrm{Pe}=O(\varepsilon), \mathrm{Da}=O\left(\varepsilon^{2}\right)$ for each sink distribution. This process elucidates whether the dominant corrections to the homogenized mean concentration profile arise from discreteness of the sinks (as is the case for normally perturbed sinks with $\sigma \ll 1$ ) or from spatial disorder (in the uniformly-random case). Likewise, our results demonstrate when fluctuations in the concentration become comparable in size to the mean, signifying a breakdown of the expansion. Interestingly, for the present problem the homogenized approximation holds for $\mathrm{Da} \ll 1$ (with $\mathrm{Pe} \ll 1$ ), breaking down in region [U] as $\mathrm{Da}$ approaches unity both in the strictly periodic case (when the dominant relative error is $O\left(\mathrm{Da}^{1 / 2}\right)$, see Table 1 ) and the uniformly random case (when the dominant relative error is $O\left(\mathrm{Da}^{1 / 4}\right)$, see Table 2).

Our results provide evidence that for a periodic sink array, the classical method employing a twoscale expansion and a unit-cell average (summarized in Appendix B) fails to account accurately for higher-order corrections in the concentration field. The alternative method presented here neither assumes that the concentration explicitly depends on two spatial variables nor that it is periodic across unit cells, 
and it recovers terms missing in the classical approach that improve agreement with numerical simulations (up to a given order of $\varepsilon$ ), as illustrated in Figure 3. Limitations of the two-scale method in dealing with boundary conditions have been noted previously by Pavliotis \& Stuart (2008).

When sink locations are weakly perturbed, the concentration field has a spatial correlation structure that extends across the entire domain, even though the perturbations to the sink locations are independent. Our Green's function-based approach provides an explicit prediction of these correlations in terms of a non-local combination of $G$ and the leading-order concentration profile $C_{\mathrm{H}}$ which agrees well with simulations in a large region of $(\mathrm{Pe}, \mathrm{Da})$-parameter space (Figure 4). In the present problem the first corrections to the mean concentration that result from weakly disordered sinks are smaller in magnitude than the corrections due to discreteness (arising in the periodic problem), provided $\sigma \ll 1$. This is confirmed by comparison with simulations of the ensemble averaged residual $\langle r(x)\rangle$ (see Figure 5). In our previous study of the case in which sink strength, rather than sink location, was disordered, we found that fluctuation magnitudes could be greatest towards the downstream end of the domain when advection was strong (Russell et al., 2016), unlike the pattern of disorder shown in Figure 4.

Strongly disordered sink locations were modelled using a uniform distribution $\mathscr{U}\left(0, \varepsilon^{-1}\right)$ and labelled in ascending order from the inlet to the outlet of the domain. The location of the $j$-th sink, $\xi_{j}$, is therefore the $j$-th order statistic of the uniform distribution out of $N$. Before relabelling, the locations are independent random variables but the sorting introduces correlations between the sink locations. Using results on order statistics, we derived predictions of the long-range correlations in the concentration induced by sink disorder, which again agree well with simulations (see Figure 6). Unlike weakly perturbed sinks, we found that strong disorder has a significant effect on the mean concentration, contributing at $O(\varepsilon)$ in the distinguished limit (Table 2). This is an order of magnitude larger than the oscillatory terms arising from a periodic array, and we therefore compare the mean stochastic corrections with the sample mean of the residual $\langle r(x)\rangle$ from simulations, rather than with $\operatorname{Da} f(x-k) C_{\mathrm{H}}(x)$ as before (see Figure 7). Our estimate of the perturbation to the mean concentration induced by disorder shows excellent quantitative accuracy for smaller values of Da; the relative error in the mean is $O\left(\mathrm{Da}^{1 / 2}\right)$ as uptake becomes stronger, and this grows as Da increases towards unity. Unlike the case of variable sink strength (Russell et al., 2016), here the correction to the mean due to disordered sink locations can change sign across the domain.

In practical applications it can be important to understand not only large-scale concentration distributions across a region but also small-scale variations across unit cells. In the placenta, for example, transfer between fetal and maternal circulation takes place at the lengthscale of individual terminal villi, where individual fetal capillary loops within a branch come into close proximity to maternal blood outside the branch (Erlich et al., 2018). The size of solute fluctuations across an individual branch can be expected to influence the transport across the surface of the branch. Given the high degree of spatial disorder in branches (Chernyavsky et al., 2011; Erlich et al., 2018), the fluctuations associated with spatial disorder (reflected by the standard deviation of $\hat{C}_{b}$ in Table 2) deserve particular attention, particularly if there are correlations between the orientation of capillary loops within the villous branch and the position of the branch with respect to its neighbours.

There are a number of obvious extensions of the present work, for example to consider other distinguished limits in parameter space, nonlinear uptake kinetics and unsteady effects. A similar steady problem with zeroth-order kinetics was analysed in Chernyavsky et al. $(2011,2012)$ using a direct algebraic method to capture stochastic behaviour. A Green's function approach may be applicable to such cases but the effort in calculating nonlinear, nonlocal and unsteady expressions is likely to be greater than in the present case. A further important class of problems to consider involves finite-size sinks in two or more dimensions, where there has been substantial effort in deriving upscaled approximations 
for electrokinetics (Heitzinger \& Ringhofer, 2014; Schmuck \& Bazant, 2015) and reactive flow in disordered porous media (Cushman et al., 2002). The present approach should provide a useful foundation for investigations characterising the multiscale structure of stochastic flow and solute fields in higher dimensions.

\section{Acknowledgements}

We are grateful to Tobias Galla for helpful conversations. OEJ was supported by EPSRC grant EP/K037145/1.

\section{A. Numerics}

In this section we describe a hybrid method for generating realisations of the concentration profile for arbitrary sink distributions. We represent solutions of (2.4) exactly as an algebraic linear system, which we solve numerically for a given sink distribution.

We first write (2.4a) as an advection-diffusion equation between sinks and a condition in the jump in concentration gradient at each sink. Integrating in a vanishing region around sink $j$ and using continuity of the concentration, gives the jump condition

$$
\llbracket C_{x} \rrbracket_{x=\xi_{j}}=\mathrm{DaC}\left(\xi_{j}\right), \quad j=1, \ldots, N,
$$

whereas in the bulk of the domain,

$$
C_{x x}-\operatorname{Pe} C_{x}=0, \quad 0<x<\varepsilon^{-1}, \quad x \neq \xi_{j},
$$

supplemented with the boundary conditions (2.4b) and continuity of $C$ across sinks, $\llbracket C \rrbracket_{x=\xi_{j}}=0$. Integrating (A.2) twice, we find

$$
C(x)=A_{j} e^{\mathrm{Pe}\left(x-\xi_{j}\right)}+B_{j}, \quad \xi_{j} \leqslant x \leqslant \xi_{j+1}, \quad j=0, \ldots, N,
$$

where the $A_{j}, B_{j}$ are constants. The inlet and outlet boundary conditions yield

$$
B_{0}=\varepsilon / \mathrm{Pe}, \quad A_{N} e^{\mathrm{Pe}\left(\varepsilon^{-1}-\xi_{N}\right)}+B_{N}=0,
$$

respectively. Continuity of $C$ across sinks implies,

$$
A_{j}-A_{j-1} e^{\mathrm{Pe}\left(\xi_{j}-\xi_{j-1}\right)}+B_{j}-B_{j-1}=0,
$$

and the jump condition (A.1) gives,

$$
A_{j}-A_{j-1} e^{\mathrm{Pe}\left(\xi_{j}-\xi_{j-1}\right)}-\frac{\mathrm{Da}}{\mathrm{Pe}}\left(A_{j}+B_{j}\right)=0 .
$$

Eliminating $A_{j-1}$ from (A.4b) via (A.4c), gives the following sparse linear system of $2(N+1)$ algebraic equations for the $A_{j}, B_{j}$,

$$
\begin{aligned}
B_{0} & =\frac{\varepsilon}{\mathrm{Pe}}, \\
\frac{\mathrm{Da}}{\mathrm{Pe}} A_{j}+\left(1+\frac{\mathrm{Da}}{\mathrm{Pe}}\right) B_{j}-B_{j-1} & =0, \quad j=1, \ldots, N \\
\left(1-\frac{\mathrm{Da}}{\mathrm{Pe}}\right) A_{j}-e^{\mathrm{Pe}\left(\xi_{j}-\xi_{j-1}\right)} A_{j-1}-\frac{\mathrm{Da}}{\mathrm{Pe}} B_{j} & =0, \quad j=1, \ldots, N \\
A_{N} e^{\mathrm{Pe}\left(\varepsilon^{-1}-\xi_{N}\right)}+B_{N} & =0 .
\end{aligned}
$$


This is an exact representation of (2.4) but an explicit solution for the $A_{j}, B_{j}$ is not readily available. Therefore, for a given sink distribution, we find $A_{j}, B_{j}$ via a numerical solution of the linear system (A.5), and use the coefficients in the bulk solution (A.3) to reconstruct the concentration profile. An ensemble of such concentration profiles with sinks placed according to some random distribution can be used to generate sample statistics which we will use to validate theoretical predictions.

\section{B. Classical two-scale homogenization for transport past a periodic sink array}

We use a classical homogenization approach to analyse the periodic sinks problem for comparison with the method described in Sec. 4 . We begin with (2.4) with $\xi_{j}=j$, i.e.

$$
\begin{gathered}
C_{x x}-\operatorname{Pe} C_{x}=\operatorname{Da} C(x) S(x), \quad 0<x<\varepsilon^{-1}, \\
\left.\operatorname{Pe} C\right|_{x=0}-\left.C_{x}\right|_{x=0}=\varepsilon, \\
\left.C\right|_{x=\varepsilon^{-1}}=0 \\
S(x)=\sum_{i=1}^{N} \delta(x-i)
\end{gathered}
$$

Let $X=\varepsilon x$ denote a "long-range" spatial variable which takes values in $[0,1]$ across the domain. We then seek solutions of the form $C(x)=\widetilde{C}(x, \varepsilon x)$, where we expand in powers of $\varepsilon$,

$$
\widetilde{C}(x, X)=C^{(0)}(x, X)+\varepsilon C^{(1)}(x, X)+\varepsilon^{2} C^{(2)}(x, X)+\cdots,
$$

where $C^{(n)}(x, X)=O(1)$, for $n=0,1,2, \ldots$ The variables $x$ and $X$ will be treated as independent. Derivatives transform according to

$$
\frac{\mathrm{d}}{\mathrm{d} x}=\frac{\partial}{\partial x}+\varepsilon \frac{\partial}{\partial X}, \quad \frac{\mathrm{d}^{2}}{\mathrm{~d} x^{2}}=\frac{\partial^{2}}{\partial x^{2}}+2 \varepsilon \frac{\partial^{2}}{\partial x \partial X}+\varepsilon^{2} \frac{\partial^{2}}{\partial X^{2}}
$$

We investigate solutions in the distinguished limit $\mathrm{Pe}=O(\varepsilon), \mathrm{Da}=O\left(\varepsilon^{2}\right)$. Thus, we set $\mathrm{Pe}=\varepsilon p_{0}$ and $\mathrm{Da}=\varepsilon^{2} q_{0}$, where $p_{0}, q_{0}=O(1)$ as $\varepsilon \rightarrow 0$. Define the linear operator and boundary condition operator as

$$
\mathscr{L}_{x} \equiv \frac{\partial^{2}}{\partial x^{2}}, \quad \mathscr{B}_{x} C \equiv\left\{\left.C_{x}\right|_{x=0},\left.C\right|_{X=1}\right\}
$$

respectively. We have included the subscripts to distinguish these operators from those in the main text. 
Then substituting (B.2) into (B.1) and collecting terms in orders of $\varepsilon$, we obtain the following systems:

$$
\begin{aligned}
& O(1):\left\{\begin{array}{l}
\mathscr{L}_{x} C^{(0)}=0 \\
\mathscr{B}_{x} C^{(0)}=\{0,0\}
\end{array}\right. \\
& O(\varepsilon):\left\{\begin{array}{l}
\mathscr{L}_{x} C^{(1)}=-2 C_{x X}^{(0)}+p_{0} C_{x}^{(0)} \\
\mathscr{B}_{x} C^{(1)}=\left\{\left.p_{0} C^{(0)}\right|_{X=0}-\left.C_{X}^{(0)}\right|_{X=0}-1,0\right\}
\end{array}\right. \\
& O\left(\varepsilon^{2}\right):\left\{\begin{array}{l}
\mathscr{L}_{x} C^{(2)}=q_{0} C^{(0)} S(x)-2 C_{x X}^{(1)}-C_{X X}^{(0)}+p_{0}\left(C_{x}^{(1)}+C_{X}^{(0)}\right) \\
\mathscr{B}_{x} C^{(2)}=\left\{\left.p_{0} C^{(1)}\right|_{X=0}-\left.C_{X}^{(1)}\right|_{X=0}, 0\right\}
\end{array}\right. \\
& O\left(\varepsilon^{3}\right):\left\{\begin{array}{l}
\mathscr{L}_{x} C^{(3)}=q_{0} C^{(1)} S(x)-2 C_{x X}^{(2)}-C_{X X}^{(1)}+p_{0}\left(C_{x}^{(2)}+C_{X}^{(1)}\right) \\
\mathscr{B}_{x} C^{(3)}=\left\{\left.p_{0} C^{(2)}\right|_{X=0}-\left.C_{X}^{(2)}\right|_{X=0}, 0\right\}
\end{array}\right.
\end{aligned}
$$

and so on for higher orders.

We seek $x$-periodic solutions and therefore we will work in a representative unit cell by defining new coordinates, $x^{\prime} \equiv x-i$, so that $-1 / 2<x^{\prime}<1 / 2$ in each cell. Sinks are therefore situated at $x^{\prime}=0$ in each cell.

At $O(1)$, we have

$$
\begin{gathered}
C_{x^{\prime} x^{\prime}}^{(0)}=0, \\
\left.C_{x^{\prime}}^{(0)}\right|_{X=0}=0,\left.\quad C^{(0)}\right|_{X=1}=0, \\
\llbracket C^{(0)} \rrbracket_{x^{\prime}=0}=0, \quad \llbracket C_{x^{\prime}}^{(0)} \rrbracket_{x^{\prime}=0}=0, \\
x^{\prime} \text {-periodicity of } C^{(0)} .
\end{gathered}
$$

Using the periodicity condition, we therefore find that $C^{(0)}=C^{(0)}(X)$ only, along with $C^{(0)}(1)=0$.

At $O(\varepsilon)$, we have

$$
\begin{gathered}
C_{x^{\prime} x^{\prime}}^{(1)}=0, \\
\left.C_{x^{\prime}}^{(1)}\right|_{X=0}=C_{B}^{(0)},\left.\quad C^{(1)}\right|_{X=1}=0, \\
\llbracket C^{(1)} \rrbracket_{x^{\prime}=0}=0, \quad \llbracket C_{x^{\prime}}^{(1)} \rrbracket_{x^{\prime}=0}=0, \\
x^{\prime} \text {-periodicity of } C^{(1)},
\end{gathered}
$$

where $\left.C_{B}^{(0)} \equiv p_{0} C^{(0)}\right|_{X=0}-\left.C_{X}^{(0)}\right|_{X=0}-1$. Similarly to the previous order, we find that $C^{(1)}=C^{(1)}(X)$ only, with $C^{(1)}(1)=0$. Additionally, applying the boundary condition on $C^{(1)}$ at the inlet fixes the condition on $C^{(0)}$ to be $C_{B}^{(0)}=0$, or

$$
\left.p_{0} C^{(0)}\right|_{X=0}-\left.C_{X}^{(0)}\right|_{X=0}=1
$$


24 of 28

At $O\left(\varepsilon^{2}\right)$, we have

$$
\begin{gathered}
C_{x^{\prime} x^{\prime}}^{(2)}=-\left(C_{X X}^{(0)}-p_{0} C_{X}^{(0)}\right), \\
\left.C_{x^{\prime}}^{(2)}\right|_{X=0}=C_{B}^{(1)},\left.\quad C^{(2)}\right|_{X=1}=0, \\
\llbracket C^{(2)} \rrbracket_{x^{\prime}=0}=0, \quad \llbracket C_{x^{\prime}}^{(2)} \rrbracket_{x^{\prime}=0}=q_{0} C^{(0)}, \\
x^{\prime} \text {-periodicity of } C^{(2)},
\end{gathered}
$$

where $\left.C_{B}^{(1)} \equiv p_{0} C^{(1)}\right|_{X=0}-\left.C_{X}^{(1)}\right|_{X=0}$. Performing a spatial average over a unit cell and using the periodicity and jump conditions on $C^{(2)}$, we obtain the macroscopic equation for the leading order solution,

$$
C_{X X}^{(0)}-p_{0} C_{X}^{(0)}=q_{0} C^{(0)}(X)
$$

The leading order solution is

$$
C^{(0)}(X)=\frac{e^{p_{0} X / 2} \sinh [\Phi(1-X)]}{p_{0} \sinh (\Phi) / 2+\Phi \cosh (\Phi)},
$$

where $\Phi \equiv \sqrt{q_{0}+p_{0}^{2} / 4}$, consistent with (3.2).

Substituting the macroscopic equation for $C^{(0)}$ into the equation for $C^{(2)}$ and directly integrating in each half of the unit cell yields

$$
C^{(2)}=\left\{\begin{array}{lr}
-\frac{1}{2} q_{0} C^{(0)} x^{\prime 2}+a_{1} x^{\prime}+a_{2}, & -\frac{1}{2} \leqslant x^{\prime}<0, \\
-\frac{1}{2} q_{0} C^{(0)} x^{\prime 2}+b_{1} x^{\prime}+b_{2}, & 0 \leqslant x^{\prime} \leqslant \frac{1}{2}
\end{array}\right.
$$

Applying the jump conditions gives $b_{2}=a_{2}$ and $b_{1}=a_{1}+q_{0} C^{(0)}$. We then impose that $\left\langle C^{(2)}\right\rangle$ is a constant, where $\langle f\rangle=\int_{-1 / 2}^{1 / 2} f \mathrm{~d} x^{\prime}$, which gives $a_{2}=\left\langle C^{(2)}\right\rangle-\frac{1}{12} q_{0} C^{(0)}$. Periodicity then implies that $a_{1}=-\frac{1}{2} q_{0} C^{(0)}$.

It remains to find $C_{B}^{(1)}$ and $\left\langle C^{(2)}\right\rangle$ which allow $C^{(2)}$ to satisfy the global boundary conditions. We therefore find from the inlet condition that

$$
\left.C_{X^{\prime}}^{(2)}\right|_{X=0}=-\left.\frac{1}{2} q_{0} C^{(0)}\right|_{X=0}(-1)=C_{B}^{(1)}
$$

or

$$
C_{B}^{(1)}=\frac{1}{2} q_{0} C^{(0)}(0)
$$

Similarly, applying the outlet condition gives $\left\langle C^{(2)}\right\rangle=0$. Therefore the expression for the second correction is

$$
C^{(2)}=-\frac{1}{2} q_{0} C^{(0)}\left(x^{\prime 2}-\left|x^{\prime}\right|+\frac{1}{6}\right), \quad-\frac{1}{2} \leqslant x^{\prime} \leqslant \frac{1}{2}
$$


At $O\left(\varepsilon^{3}\right)$, we have

$$
\begin{gathered}
C_{x^{\prime} x^{\prime}}^{(3)}=-\left(2 C_{x^{\prime} X}^{(2)}-p_{0} C_{x^{\prime}}^{(2)}\right)-\left(C_{X X}^{(1)}-p_{0} C_{X}^{(1)}\right), \\
\left.C_{x^{\prime}}^{(3)}\right|_{X=0}=C_{B}^{(2)},\left.\quad C^{(3)}\right|_{X=1}=0, \\
\llbracket C^{(3)} \rrbracket_{x^{\prime}=0}=0, \quad \llbracket C_{x^{\prime}}^{(3)} \rrbracket_{x^{\prime}=0}=q_{0} C^{(1)}, \\
x^{\prime} \text {-periodicity of } C^{(3)},
\end{gathered}
$$

where $\left.C_{B}^{(2)} \equiv p_{0} C^{(2)}\right|_{X=0}-\left.C_{X}^{(2)}\right|_{X=0}$. Again we average over a unit cell, and find that the first correction satisfies the same macroscopic equation as the leading order solution,

$$
C_{X X}^{(1)}-p_{0} C_{X}^{(1)}=q_{0} C^{(1)}(X)
$$

Therefore the first correction is proportional to the solution at leading order and is given by

$$
C^{(1)}(X)=\frac{1}{2} q_{0} C^{(0)}(0) C^{(0)}(X)
$$

finally giving $C=C_{\mathrm{H}}(x)+\frac{1}{2} \varepsilon q_{0} C_{\mathrm{H}}(0) C_{\mathrm{H}}(x)+\varepsilon^{2} q_{0} C_{\mathrm{H}}(x) f\left(x^{\prime}\right)+O\left(\varepsilon^{3}\right)$, missing one term in (4.4) and the $O\left(\varepsilon^{2}\right)$ term in (4.6).

\section{Unit cell integration identities}

For $j=1,2, \ldots, N$,

$$
\int_{j-1 / 2}^{j+1 / 2}(y-j)^{n}[\delta(y-j)-1] \mathrm{d} y= \begin{cases}0, & n=0,1 \\ -\frac{1}{12}, & n=2\end{cases}
$$

For $k-1 / 2<x<k+1 / 2$ (i.e. $k=\lfloor x+1 / 2\rfloor$ ),

$$
\begin{gathered}
\int_{k-1 / 2}^{x}[\boldsymbol{\delta}(y-k)-1] \mathrm{d} y=H(x-k)-x+k-\frac{1}{2}, \\
\int_{x}^{k+1 / 2}[\delta(y-k)-1] \mathrm{d} y=H(k-x)+x-k-\frac{1}{2}, \\
\int_{k-1 / 2}^{x}(y-x)[\delta(y-k)-1] \mathrm{d} y=(k-x) H(x-k)+\frac{1}{2}\left(x-k+\frac{1}{2}\right)^{2}, \\
\int_{x}^{k+1 / 2}(y-x)[\delta(y-k)-1] \mathrm{d} y=(k-x) H(k-x)-\frac{1}{2}\left(x-k-\frac{1}{2}\right)^{2}, \\
\int_{k-1 / 2}^{x}(y-x)^{2}[\delta(y-k)-1] \mathrm{d} y=(k-x)^{2} H(x-k)-\frac{1}{3}\left(x-k+\frac{1}{2}\right)^{3}, \\
\int_{x}^{k+1 / 2}(y-x)^{2}[\delta(y-k)-1] \mathrm{d} y=(k-x)^{2} H(k-x)+\frac{1}{3}\left(x-k-\frac{1}{2}\right)^{3} .
\end{gathered}
$$


26 of 28

\section{Approximating sums with integrals}

Let $f(y)$ be a smooth function, except possibly at $y=k$, with $f(y)=O\left(\varepsilon^{a}\right)$ as $\varepsilon \rightarrow 0$ for some $a \in \mathbb{R}$. Additionally assume that successive derivatives of $f(y)$ fall in magnitude by a factor of $\varepsilon$; i.e. $\frac{\mathrm{d}^{n} f}{\mathrm{~d} y^{n}}=$ $O\left(\varepsilon^{a+n}\right)$. We decompose the integral $\int_{0}^{x^{-}} f(y) \mathrm{d} y$ into contributions from unit cells, centred at $y=j$, $j=1,2, \ldots, k-1$, a half-cell from $y=0$ and the remaining interval $\left[k-\frac{1}{2}, x\right]$; we split the integral $\int_{x^{+}}^{\varepsilon^{-1}} f(y) \mathrm{d} y$ similarly. Then

$$
\begin{aligned}
& \int_{0}^{x^{-}} f(y) \mathrm{d} y=\int_{0}^{1 / 2} f(y) \mathrm{d} y+\sum_{j=1}^{k-1} \int_{j-1 / 2}^{j+1 / 2} f(y) \mathrm{d} y+\int_{k-1 / 2}^{x^{-}} f(y) \mathrm{d} y \\
& \int_{x^{+}}^{\mathcal{E}^{-1}} f(y) \mathrm{d} y=\int_{x^{+}}^{k+1 / 2} f(y) \mathrm{d} y+\sum_{j=k+1}^{N} \int_{j-1 / 2}^{j+1 / 2} f(y) \mathrm{d} y+\int_{\mathcal{E}^{-1}-1 / 2}^{\varepsilon^{-1}} f(y) \mathrm{d} y
\end{aligned}
$$

Taylor expanding around $y=0$ and $y=\varepsilon^{-1}$ for the half-cells, around the centre of each unit cell and $y=x^{ \pm}$for the intervals from and up to $x$,

$$
\begin{aligned}
& f(y)=f(0)+y f_{y}(0)+\frac{1}{2} y^{2} f_{y y}(0)+\cdots \\
& f(y)=f(j)+(y-j) f_{y}(j)+\frac{1}{2}(y-j)^{2} f_{y y}(j)+\cdots \\
& f(y)=f\left(x^{ \pm}\right)+(y-x) f_{y}\left(x^{ \pm}\right)+\frac{1}{2}(y-x)^{2} f_{y y}\left(x^{ \pm}\right)+\cdots \\
& f(y)=f\left(\varepsilon^{-1}\right)+\left(y-\varepsilon^{-1}\right) f_{y}\left(\varepsilon^{-1}\right)+\frac{1}{2}\left(y-\varepsilon^{-1}\right)^{2} f_{y y}\left(\varepsilon^{-1}\right)+\cdots
\end{aligned}
$$

Integrating each term in (D.2),

$$
\begin{aligned}
\int_{0}^{1 / 2} f(y) \mathrm{d} y & =\frac{1}{2} f(0)+\frac{1}{8} f_{y}(0)+\frac{1}{48} f_{y y}(0)+\cdots, \\
\int_{j-1 / 2}^{j+1 / 2} f(y) \mathrm{d} y & =f(j)+\frac{1}{24} f_{y y}(j)+\cdots \\
\int_{k-1 / 2}^{x^{-}} f(y) \mathrm{d} y & =f\left(x^{-}\right)\left(x-k+\frac{1}{2}\right)-\frac{1}{2} f_{y}\left(x^{-}\right)\left(x-k+\frac{1}{2}\right)^{2}+\frac{1}{6} f_{y y}\left(x^{-}\right)\left(x-k+\frac{1}{2}\right)^{3}+\cdots, \\
\int_{x^{+}}^{k+1 / 2} f(y) \mathrm{d} y & =f\left(x^{+}\right)\left(k-x+\frac{1}{2}\right)+\frac{1}{2} f_{y}\left(x^{+}\right)\left(k-x+\frac{1}{2}\right)^{2}+\frac{1}{6} f_{y y}\left(x^{+}\right)\left(k-x+\frac{1}{2}\right)^{3}+\cdots, \\
\int_{\varepsilon^{-1}-1 / 2}^{\varepsilon^{-1}} f(y) \mathrm{d} y & =\frac{1}{2} f\left(\varepsilon^{-1}\right)-\frac{1}{8} f_{y}\left(\varepsilon^{-1}\right)+\frac{1}{48} f_{y y}\left(\varepsilon^{-1}\right)+\cdots
\end{aligned}
$$

Using (D.3) in (D.1) and rearranging, we have

$$
\begin{aligned}
\sum_{j \neq k} f(j)= & \left(\int_{0}^{x^{-}}+\int_{x^{+}}^{\varepsilon^{-1}}\right) f(y) \mathrm{d} y \\
& -\frac{1}{2}\left[f(0)+f\left(\varepsilon^{-1}\right)\right]-\left[f\left(x^{+}\right)\left(k-x+\frac{1}{2}\right)+f\left(x^{-}\right)\left(x-k+\frac{1}{2}\right)\right] \\
& -\frac{1}{8}\left[f_{y}(0)-f_{y}\left(\varepsilon^{-1}\right)\right]-\frac{1}{24} \sum_{j \neq k} f_{y y}(j)-\frac{1}{2}\left[f_{y}\left(x^{+}\right)\left(k-x+\frac{1}{2}\right)^{2}-\frac{1}{2} f_{y}\left(x^{-}\right)\left(x-k+\frac{1}{2}\right)^{2}\right] \\
& +\cdots
\end{aligned}
$$




\section{E. Averaging unit-cell fluctuations}

We seek $F(x)=\langle f(x-k-\phi)\rangle$ where $k=\left\lfloor x+\frac{1}{2}\right\rfloor$ and $f(x)=-\frac{1}{2}\left(x^{2}-|x|+\frac{1}{6}\right)$ in $|x| \leqslant \frac{1}{2}$ (and is zero otherwise), with $\phi \sim \mathscr{N}\left(0, \sigma^{2}\right)$, with $\sigma \ll 1$. We restrict attention to the cell $k=0$. Where $f$ varies smoothly with respect to $x$ (away from $x=0$ ), $F(x)=f(x)+O\left(\sigma^{2}\right)$. However the spike in $f$ near $x=0$ is smoothed more dramatically. Let $g(\phi)=\left(2 \pi \sigma^{2}\right)^{-1 / 2} \exp \left(-\phi^{2} /\left(2 \sigma^{2}\right)\right)$ be the probability density of the shift $\phi$. Then for $x$ in a region of width $O(\sigma)$ near the origin,

$$
\begin{aligned}
F(x) & =\int_{-\infty}^{\infty}\left(-\frac{1}{12}+\frac{1}{2}|x-\phi|+\ldots\right) g(\phi) \mathrm{d} \phi+O\left(\sigma^{2}\right) \\
& =-\frac{1}{12}+\int_{-\infty}^{x} \frac{1}{2}(x-\phi) g(\phi) \mathrm{d} \phi+\int_{x}^{\infty} \frac{1}{2}(\phi-x) g(\phi) \mathrm{d} \phi+\ldots \\
& =-\frac{1}{12}+\frac{\sigma}{\sqrt{2 \pi}} \exp \left(-\frac{x^{2}}{2 \sigma^{2}}\right)+\frac{x}{2} \operatorname{erf}\left(\frac{x}{\sigma \sqrt{2}}\right)+\ldots
\end{aligned}
$$

The outer limit of this approximation, for $x \gg \sigma$, gives $F \approx-\frac{1}{12}+\frac{1}{2}|x|+O\left(\sigma^{2}\right)$, matching with the region where $f$ varies smoothly. For $x \ll \sigma$,

$$
F \approx-\frac{1}{12}+\frac{\sigma}{\sqrt{2 \pi}}+\frac{1}{2 \sigma} \frac{x^{2}}{\sqrt{2 \pi}}+O\left(\sigma^{2}\right) .
$$

Thus small normal perturbations of sink locations reduce the amplitude of unit-cell fluctuations from $\left(-\frac{1}{12}, \frac{1}{24}\right)$ (the range of $f$ ) to $\left(-\frac{1}{12}+\sigma / \sqrt{2 \pi}, \frac{1}{24}\right)$ (the range of $F$, with error $O\left(\sigma^{2}\right)$ ). Stronger disorder suppresses the range completely: when $\phi \sim \mathscr{U}\left(-\frac{1}{2}, \frac{1}{2}\right)$,

$$
F(x)=\int_{-1 / 2}^{1 / 2} \sum_{k} f(x-k-\phi) \mathrm{d} \phi=\int_{x-1 / 2}^{x+1 / 2} \sum_{k} f(u-k) \mathrm{d} u=0 .
$$

\section{REFERENCES}

Ahsanullah, M., Nevzorov, V. B. \& Shakil, M. (2013) An Introduction to Order Statistics. Springer.

Bal, G. (2011) Convergence to homogenized or stochastic partial differential equations. App. Math. Res. eXpress, 2011(2), 215-241.

Bruna, M. \& Chapman, S. J. (2015) Diffusion in spatially varying porous media. SIAM J. Appl. Math., 75(4), 1648-1674.

Burridge, R. \& Keller, J. B. (1981) Poroelasticity equations derived from microstructure. J. Acoust. Soc. Am., 70(4), 1140-1146.

Chernyavsky, I., Jensen, O. \& Leach, L. (2010) A mathematical model of intervillous blood flow in the human placentone. Placenta, 31(1), 44-52.

Chernyavsky, I. L., Dryden, I. L. \& Jensen, O. E. (2012) Characterizing the multiscale structure of fluctuations of transported quantities in a disordered medium. IMA J. Appl. Math., 77(5), 697-725.

Chernyavsky, I. L., Leach, L., Dryden, I. L. \& Jensen, O. E. (2011) Transport in the placenta: homogenizing haemodynamics in a disordered medium. Phil. Trans. R. Soc. A, 369(1954), 4162-4182.

Chunsheng, M. (1992) Moments of functions of order statistics. Statistics \& Prob. Lett., 15(1), 57-62.

Cushman, J. H., Bennethum, L. S. \& Hu, B. X. (2002) A primer on upscaling tools for porous media. Adv. Water Res., 25(8), 1043-1067.

David, H. A. \& Nagaraja, H. N. (2003) Order Statistics. John Wiley \& Sons, Inc., 3 edition. 
Davit, Y., Bell, C. G., Byrne, H. M., Chapman, L. A., Kimpton, L. S., Lang, G. E., Leonard, K. H., Oliver, J. M., Pearson, N. C., Shipley, R. J. et al. (2013) Homogenization via formal multiscale asymptotics and volume averaging: How do the two techniques compare?. Adv. Water Res., 62, 178-206.

Erian, F., Corrsin, S. \& Davis, S. (1977) Maternal, placental blood flow: a model with velocity-dependent permeability. J. Biomech., 10(11-12), 807-814.

Erlich, A., Pearce, P., Plitman Mayo, R., Jensen, O. E. \& Chernyavsky, I. L. (2018) Physical and geometric determinants of transport in feto-placental microvascular networks. arXiv:1809.00749.

Heitzinger, C. \& Ringhofer, C. (2014) Multiscale modeling of fluctuations in stochastic elliptic PDE models of nanosensors. Commun. Math. Sci, 12(3), 401-421.

Jensen, O. E. \& Chernyavsky, I. L. (2019) Blood Flow and Transport in the Human Placenta. Ann. Rev. Fluid Mech., 51, 25-47.

Le Bris, C. (2014) Homogenization theory and multiscale numerical approaches for disordered media: some recent contributions. ESAIM: Proc. Surveys, 45, 18-31.

Pavliotis, G. \& Stuart, A. (2008) Multiscale methods: averaging and homogenization. Springer Science \& Business Media.

Rubinstein, J. \& Torquato, S. (1989) Flow in random porous media: mathematical formulation, variational principles, and rigorous bounds. J. Fluid Mech., 206, 25-46.

Russell, M. J., Jensen, O. E. \& Galla, T. (2016) Stochastic transport in the presence of spatial disorder: Fluctuationinduced corrections to homogenization. Phys. Rev. E, 94(4), 042121.

Schmuck, M. \& Bazant, M. Z. (2015) Homogenization of the Poisson-Nernst-Planck equations for ion transport in charged porous media. SIAM J. Appl. Math., 75(3), 1369-1401.

Wood, B. D. \& Valdés-Parada, F. J. (2013) Volume averaging: Local and nonlocal closures using a Green's function approach. Adv. Water Res., 51, 139-167. 